\title{
On the Galois structure of arithmetic cohomology II: ray class groups
}

\author{
By David Burns and Asuka Kumon
}

(Received Sep. 25, 2015)

(Revised July 27, 2016)

\begin{abstract}
We investigate the explicit Galois structure of ray class groups. We then derive consequences of our results concerning both the validity of Leopoldt's Conjecture and the existence of families of explicit congruence relations between the values of Dirichlet $L$-series at $s=1$.
\end{abstract}

\section{Introduction.}

The primary aim of this article is to investigate aspects of the explicit Galois structure of a natural family of ray class groups of number fields. This subject has a rather long history but aside from any intrinsic interest it may have there are also two ways in which it can have important consequences.

Firstly, such Galois structures are closely linked to the validity, or otherwise, of Leopoldt's Conjecture. In this context they have already been much studied in the literature, both in relatively simple cases (see, for example, Miki and Sato [22]) and in more involved Iwasawa-theoretic contexts (see, for example, Khare and Wintenberger $[\mathbf{1 8}])$.

Secondly, ray class groups arise in the cohomology of complexes that occur in the formulation of the natural leading term conjectures for both $p$-adic and complex valued equivariant $L$-series that have been studied in recent years and, via this interpretation, explicit structural information on ray class groups directly translates into explicit congruence relations between such leading terms.

To study this problem we combine aspects of the approach developed by the first author in [5], [6] to investigate the explicit Galois structure of arithmetic cohomology groups together with the approach used by Macias Castillo and the first author in [8] to prove a natural interpretation of the validity of Leopoldt's Conjecture in terms of the cohomological-triviality, as Galois modules, of a natural family of ray class groups.

In particular, in our first result we shall give a new interpretation of Leopoldt's Conjecture in terms of the basic properties of certain canonical 'étale' and 'cyclotomic' Yoneda extension classes that we shall introduce (in Section 2.2).

We then prove some detailed results about the explicit Galois structure of ray class groups and discuss consequences of these results for the validity of Leopoldt's Conjecture (see, for example, Theorem 2.14 and Remark 2.15).

2010 Mathematics Subject Classification. Primary 11G35; Secondary 11R33, 11R34.

Key Words and Phrases. ray class groups, Galois structure, Leopoldt's Conjecture, Dirichlet $L$ series. 
Finally, as a concrete application of this general approach, we combine our results on Yoneda extensions and explicit Galois structures to prove several new results concerning the arithmetic of cyclotomic fields of conductor a power of any prime $p$ that validates Vandiver's Conjecture.

These results include establishing families of explicit congruence relations between the values at $s=1$ of the Dirichlet $L$-series associated to characters of $p$-power conductor after normalisation by a natural $p$-adic logarithmic resolvent if the character is odd and by a canonical $\mathcal{L}$-invariant (that is defined by comparing archimedean and $p$-adic logarithm maps) if the character is even.

We also prove a natural analogue of the classical fact that Stickelberger ideals account for all Galois relations in the ideal class groups of fields of $p$-power conductor (see Washington [30, Section 10.3]) in which ideal class groups are replaced by the torsion subgroups of ray class groups and Stickelberger elements by the values at $s=1$ of Dirichlet $L$-series of even characters (normalised by the canonical $\mathcal{L}$-invariants). For more details of these results see Theorem 2.17 and Remark 2.18.

For convenience of the reader, we collect together the statements of all of our main results in Section 2. These results are then proved in the remainder of the paper.

The authors are grateful to Daniel Macias Castillo for helpful discussions and to the referee for a very careful reading of the article that led to important corrections and improvements of our results.

\section{Statement of the main results.}

At the outset we fix an odd prime $p$ and write $\mathbb{F}_{p}$ for the field of cardinality $p$.

For any abelian group $M$ we write $M_{[p]}$ for its subgroup of elements of order dividing $p$ and $M_{\text {tor }}$ for its torsion subgroup and set $\bar{M}:=M / M_{\text {tor }}$.

If $M$ is a finitely generated $\mathbb{Z}_{p}$-module, then we set $\operatorname{rk}_{\mathbb{Z}_{p}}(M):=\operatorname{dim}_{\mathbb{Q}_{p}}\left(\mathbb{Q}_{p} \otimes_{\mathbb{Z}_{p}} M\right)$ and write $\operatorname{rk}_{p}(M)$ for the $p$-rank $\operatorname{dim}_{\mathbb{F}_{p}}(M / p)=\operatorname{dim}_{\mathbb{F}_{p}}\left(M_{[p]}\right)+\operatorname{rk}_{\mathbb{Z}_{p}}(M)$ of $M$. In this case we also often abbreviate $\mathbb{Q}_{p} \otimes_{\mathbb{Z}_{p}} M$ to $\mathbb{Q}_{p} \cdot M$.

For any commutative noetherian ring $R$ we write $D(R)$ for the derived category of $R$-modules and $D^{\text {perf }}(R)$ for the full triangulated subcategory of $D(R)$ comprising 'perfect' complexes (that is, complexes isomorphic in $D(R)$ to a bounded complex of finitely generated projective $R$-modules).

We denote the Galois group of a Galois extension of fields $F / E$ by $G_{F / E}$.

\subsection{Some general field notation.}

We fix an extension of number fields $L / K$. We write $L^{\text {cyc }}$ for the cyclotomic $\mathbb{Z}_{p^{-}}$ extension of $L$ and set

$$
\Gamma_{L}:=G_{L^{\mathrm{cyc}} / L}
$$

We write $\Sigma_{p}$ for the set of $p$-adic places of $K$. We fix a finite set of places $\Sigma$ of $K$ that contains $\Sigma_{p}$ and write $M_{L}^{\Sigma}$ for the maximal abelian pro- $p$ extension of $L$ that is unramified outside all places above those in $\Sigma$.

We then set 


$$
A_{L}^{\Sigma}:=G_{M_{L}^{\Sigma} / L} \quad \text { and } \quad B_{L}^{\Sigma}:=G_{M_{L}^{\Sigma} / L^{c y c}} .
$$

If $L / K$ is Galois, then we regard both of these groups as $\mathbb{Z}_{p}\left[G_{L / K}\right]$-modules via the natural conjugation action of $G_{L / K}$.

In the special case that $\Sigma=\Sigma_{p}$ we often abbreviate $M_{L}^{\Sigma_{p}}, A_{L}^{\Sigma_{p}}$ and $B_{L}^{\Sigma_{p}}$ to $M_{L}^{p}, A_{L}^{p}$ and $B_{L}^{p}$ respectively.

\subsection{The cyclotomic and étale extension classes.}

For any finite group $G$, any non-negative integer $n$ and $\mathbb{Z}_{p}[G]$-modules $M$ and $N$ we write $\operatorname{YExt}_{G}^{n}(N, M)$ for the group which classifies Yoneda $n$-extensions of $N$ by $M$.

2.2.1. Then, after fixing a topological generator $\gamma_{L}$ of $G_{L^{\text {cyc }} / L}$, the canonical group extension

$$
0 \rightarrow B_{L}^{\Sigma} \rightarrow A_{L}^{\Sigma} \rightarrow \Gamma_{L} \rightarrow 0
$$

gives rise to an element $c_{L / K}^{\Sigma, \gamma_{L}}$ of the group

$$
\operatorname{YExt}_{G_{L / K}}^{1}\left(\Gamma_{L}, B_{L}^{\Sigma}\right) \cong \operatorname{YExt}_{G_{L / K}}^{1}\left(\mathbb{Z}_{p}, B_{L}^{\Sigma}\right) \cong H^{1}\left(G_{L / K}, B_{L}^{\Sigma}\right)
$$

where the first isomorphism is induced by the choice of $\gamma_{L}$ and the second isomorphism is canonical. and $\gamma_{L}$.

We refer to the element $c_{L / K}^{\Sigma, \gamma_{L}}$ as the 'cyclotomic extension class' associated to $L / K, \Sigma$

2.2.2. We next write $\mathcal{O}_{L, \Sigma}$ for the subring of $L$ comprising elements that are integral at all non-archimedean places that do not lie above a place in $\Sigma$. We regard the Tate module $\mathbb{Z}_{p}(1)$ as an étale pro-sheaf on $\operatorname{Spec}\left(\mathcal{O}_{L, \Sigma}\right)$ in the natural way and write $R \Gamma_{c \text {,ét }}\left(\mathcal{O}_{L, \Sigma}, \mathbb{Z}_{p}(1)\right)$ for the associated complex of compactly supported étale cohomology.

If $L / K$ is Galois and $\Sigma$ contains all places that ramify in $L / K$, then the action of $G_{L / K}$ on $\mathcal{O}_{L, \Sigma}$ means that $R \Gamma_{c, \text { ét }}\left(\mathcal{O}_{L, \Sigma}, \mathbb{Z}_{p}(1)\right)$ is naturally an object of $D\left(\mathbb{Z}_{p}\left[G_{L / K}\right]\right)$.

In addition, since the truncation $\tau^{\geq 2} R \Gamma_{c \text {,ét }}\left(\mathcal{O}_{L, \Sigma}, \mathbb{Z}_{p}(1)\right)$ of $R \Gamma_{c \text {,ét }}\left(\mathcal{O}_{L, \Sigma}, \mathbb{Z}_{p}(1)\right)$ in degrees greater than or equal to two is acyclic outside degrees two and three and has cohomology in these degrees which identifies with $A_{L}^{\Sigma}$ and $\mathbb{Z}_{p}$ respectively (see Lemma 3.1 below), it gives rise in this case to a canonical element $c_{L / K}^{\Sigma \text {,ét }}$ of

$$
\operatorname{YExt}_{G_{L / K}}^{2}\left(\mathbb{Z}_{p}, A_{L}^{\Sigma}\right) \cong H^{2}\left(G_{L / K}, A_{L}^{\Sigma}\right) .
$$

We refer to $c_{L / K}^{\Sigma, \text { ét }}$ as the 'étale extension class' associated to $L / K$ and $\Sigma$.

REMARK 2.1. In [8, Theorem 3.1(ii)] it is shown that the Shafarevic-Weil Theorem gives an alternative, and more concrete, description of the extension class $c_{L / K}^{\Sigma \text {,ét }}$.

2.2.3. Our first main result gives an explicit reinterpretation of the validity of Leopoldt's Conjecture at $p$ in terms of the extension classes $c_{L / K}^{\Sigma, \gamma_{L}}$ and $c_{L / K}^{\Sigma \text {,ét }}$.

THEOREM 2.2. The following conditions are equivalent. 
(i) Leopoldt's Conjecture is valid at $p$.

(ii) For all finite Galois extensions of number fields $L / K$ and all finite sets of places $\Sigma$ of $K$ that contain all places that are either archimedean, $p$-adic or ramify in $L / K$, the complex $\tau^{\geq 2} R \Gamma_{c \text {,ét }}\left(\mathcal{O}_{L, \Sigma}, \mathbb{Z}_{p}(1)\right)$ belongs to $D^{\text {perf }}\left(\mathbb{Z}_{p}\left[G_{L / K}\right]\right)$.

(iii) For all $L / K$ and $\Sigma$ as in claim (ii) one has both

(a) $c_{L / K}^{\Sigma, \gamma_{L}}$ generates $H^{1}\left(G_{L / K}, B_{L}^{\Sigma}\right)$, and

(b) $c_{L / K}^{\Sigma, \text { ét }}$ generates $H^{2}\left(G_{L / K}, A_{L}^{\Sigma}\right)$ and has order $\left|G_{L / K}\right|$.

REMARK 2.3. The proof of Theorem 2.2 given in Section 3.2 below shows that claim (iii) is equivalent to asserting that the $G_{L / K}$-module $A_{L}^{\Sigma}$ is a class module with fundamental class $c_{L / K}^{\Sigma \text {,ét }}$ (as defined, for example, in [24, Chapter III, Definition (3.1.3)]). This statement constitutes a natural $p$-adic analogue of the fact that the $\Sigma$-idele class group of $L$ is a class module for $G_{L / K}$.

In Section 3.3 we will show that Theorem 2.2 has the following consequence.

COROLlary 2.4. Let $L / K$ be a finite Galois extension of number fields and $\Sigma$ a finite set of places of $K$ that contains all places that are either archimedean, $p$-adic or ramify in $L / K$. If $L$ validates Leopoldt's Conjecture at $p$, then the following conditions are equivalent.

(i) $c_{L / K}^{\Sigma, \gamma_{L}}$ vanishes (and so the canonical extension (1) splits).

(ii) The $G_{L / K}$-module $B_{L}^{\Sigma}$ is cohomologically-trivial.

(iii) Let $P$ be a Sylow p-subgroup of $G_{L / K}$ and set $F:=L^{P}$. Then one of the following conditions is satisfied:

(a) $L$ is contained in $F^{\text {cyc }}$;

(b) $L$ is disjoint from $F^{\mathrm{cyc}}, P$ is cyclic and for each non-trivial subgroup $C$ of $P$, with $E:=L^{C}$, the maximal abelian extension of $E^{\mathrm{cyc}}$ in $M_{L}^{\Sigma}$ is equal to $M_{E}^{\Sigma}$ and the transfer map from $A_{E}^{\Sigma}:=G_{M_{E}^{\Sigma} / E}=G_{M_{L}^{\Sigma} / E}^{\mathrm{ab}}$ to $G_{M_{L}^{\Sigma} / L}^{\mathrm{ab}}=G_{M_{L}^{\Sigma} / L}=$ : $A_{L}^{\Sigma}$ is injective upon restriction to the torsion subgroup of $G_{M_{E}^{\Sigma} / L^{\mathrm{cyc}}}$.

REMARK 2.5. One can show that, irrespective of any hypothesis on Leopoldt's Conjecture, if $L$ is contained in $K^{\mathrm{cyc}}$, then $c_{L / K}^{\Sigma, \gamma_{L}}$ vanishes (see Lemma 3.3(iii)).

REMARK 2.6. If $K$ is totally real and $L$ is a subfield of $K^{\text {cyc }}$ that validates Leopoldt's Conjecture, then $B_{L}^{\Sigma}$ is finite and Corollary 2.4 implies that either $B_{L}^{\Sigma}$ is trivial or $\left|B_{L}^{\Sigma}\right|>[L: K]$ (see Lemma 3.7). In the case that $K$ is generated over $\mathbb{Q}$ by a primitive $p$-th root of unity a stronger version of this result is proved in Theorem 2.17 below. 


\subsection{The structure of $\overline{A_{L}^{\Sigma}}$ in cyclic $p$-extensions.}

To discuss Galois structures in greater detail, in this section we consider a cyclic $p$-power degree Galois extension of number fields $L / K$ and a finite set of places $\Sigma$ of $K$ that contains all places that are either archimedean, $p$-adic or ramify in $L / K$.

For each intermediate field $F$ of $L / K$ we set

$$
T_{L / F}^{\Sigma}:=\operatorname{cok}\left(G_{M_{L}^{\Sigma} / L, \text { tor }} \rightarrow G_{M_{F}^{\Sigma} / L, \text { tor }}\right),
$$

where the arrow denotes the natural restriction map. This is a finite group and is naturally isomorphic to the torsion subgroup of $G_{\left(L^{\max } \cap M_{F}^{\Sigma}\right) / L}$ where we write $L^{\max }$ for the maximal $\mathbb{Z}_{p}$-power extension of $L$ (see Lemma 4.3 below).

We write $r_{F}$ for the number of complex places of $F$ and $\delta_{F}$ for the $p$-adic 'Leopoldt defect' of $F$. We recall that $\delta_{F}$ is a non-negative integer which vanishes if and only if Leopoldt's Conjecture is valid for $F$ at $p$ and that

$$
\operatorname{rk}_{\mathbb{Z}_{p}}\left(B_{F}^{\Sigma}\right)=\operatorname{rk}_{\mathbb{Z}_{p}}\left(A_{F}^{\Sigma}\right)-1=r_{F}+\delta_{F}
$$

(cf. [24, (10.3.7) Corollary]).

The following result is proved in Section 4.2.

THEOREM 2.7. Let $L / K$ be a cyclic extension of number fields of degree $p^{n}$ and $\Sigma$ a finite set of places of $K$ that contains all places that are either archimedean, $p$-adic or ramify in $L / K$. For each integer $i$ with $0 \leq i \leq n$ let $L_{i}$ denote the unique field with $K \subseteq L_{i} \subseteq L$ and $\left[L: L_{i}\right]=p^{i}$.

Then the $\mathbb{Z}_{p}\left[G_{L / K}\right]$-lattice $\overline{A_{L}^{\Sigma}}$ is determined up to isomorphism (in a sense to be made precise in Section 4 below) by $r_{K}$, the integers $\delta_{L_{i}}$ for each $i$ with $0 \leq i \leq n$ and the diagrams of finite groups

$$
\left\{\begin{array}{l}
T_{L / L_{1}}^{\Sigma} \rightarrow T_{L / L_{2}}^{\Sigma} \rightarrow \cdots \cdots \rightarrow T_{L / L_{n}}^{\Sigma} \\
T_{L / L_{1}}^{\Sigma} \leftarrow T_{L / L_{2}}^{\Sigma} \leftarrow \cdots \cdots \leftarrow T_{L / L_{n}}^{\Sigma}
\end{array}\right.
$$

Here each homomorphism $T_{L / L_{i}}^{\Sigma} \rightarrow T_{L / L_{i+1}}^{\Sigma}$ is induced by the natural restriction map $G_{M_{L_{i}}^{\Sigma} / L} \rightarrow G_{M_{L_{i+1}}^{\Sigma} / L}$ and each homomorphism $T_{L / L_{i+1}}^{\Sigma} \rightarrow T_{L / L_{i}}^{\Sigma}$ by the map $G_{M_{L_{i+1}}^{\Sigma} / L} \rightarrow$ $G_{M_{L_{i}}^{\Sigma} / L}$ which sends $x$ to $\sum_{c \in G_{L_{i} / L_{i+1}}} c(\tilde{x})$ where $\tilde{x}$ is any lift of $x$ to $G_{M_{L_{i}}^{\Sigma} / L}$ and we use the natural conjugation action of $G_{L_{i} / L_{i+1}}$ on $G_{M_{L_{i}}^{\Sigma} / L}$.

REMARK 2.8. A key ingredient in the proof of Theorem 2.7 is provided by a representation theoretic result of Yakovlev [31]. With a little more effort (and by using the results of $[\mathbf{3 2}]$ rather than $[\mathbf{3 1}]$ ), all of the results discussed here extend to the setting of Galois extensions $L / K$ for which only the Sylow $p$-subgroups of $G_{L / K}$ are assumed to be cyclic. However, the essential ideas are the same and so, for simplicity, we prefer not to deal with this more general case.

REMARK 2.9. The groups $A_{L, \text { tor }}^{\Sigma}$ have been extensively studied in the literature (see, in particular, the computations of Hemard in [16]) and using these results one can 
often explicitly compute the groups $T_{L / F}^{\Sigma}$ that occur in Theorem 2.7. For an example of such a computation see the proof of Lemma 6.1 below.

REmark 2.10. A $\mathbb{Z}_{p}[G]$-module is said to be a 'permutation module' if it is isomorphic to a direct sum $\bigoplus_{H \leq G} \mathbb{Z}_{p}[G / H]^{n_{H}}$ for suitable non-negative integers $n_{H}$. The proof of Theorem 2.7 implies $\overline{A_{L}^{\Sigma}}$ is a permutation $\mathbb{Z}_{p}[G]$-module if and only if $T_{L / E}^{\Sigma}=0$ for all intermediate fields $E$ of $L / K$ (see Remark 4.2). In addition, $T_{L / E}^{\Sigma}=0$ if $A_{E \text {,tor }}^{\Sigma}=0$ and Gras has characterised, assuming Leopoldt's Conjecture, those $E$ with $A_{E \text {,tor }}^{p}=0$ (see [13, Theorem I 2, Corollary 1]). It is also possible, and useful, to characterise the vanishing of $T_{L / E}^{\Sigma}$ in terms of ' $\mathbb{Z}_{p}$-extendable' extensions (see Section 4.3).

REMARK 2.11. If $L / K$ is a cyclic extension of degree either $p$ or $p^{2}$, then the indecomposable $\mathbb{Z}_{p}\left[G_{L / K}\right]$-lattices have been classified explicitly by Diederichsen [10] and Heller and Reiner [14] and these classifications can be used to give a much more explicit version of Theorem 2.7. If $L / K$ has degree $p$ such an analysis is effectively carried out by Miki and Sato in [22] (see the proof of Proposition 5.1 below for more details). However, if $L / K$ has degree $p^{2}$ the analysis is much more involved and is discussed in the upcoming thesis of the second author.

Theorem 2.7 has an interesting consequence concerning the multiplicities with which indecomposable modules occur as direct summands of the lattices $\overline{A_{L}^{p}}$.

To state this result we write $C_{n}$ for each non-negative number $n$ for the cyclic group of order $p^{n}$. For each non-negative integer $m$ with $m \leq n$ we regard $C_{m}$ as a quotient of $C_{n}$ in the obvious way and we then fix a set $\mathrm{IM}_{p}^{n}$ of representatives of the isomorphism classes of all indecomposable $\mathbb{Z}_{p}\left[C_{n}\right]$-lattices that are not isomorphic to $\mathbb{Z}_{p}\left[C_{m}\right]$ for any $m$ with $0 \leq m \leq n$.

It is known that $\operatorname{IM}_{p}^{1}$ can be taken as the singleton $\left\{\mathbb{Z}_{p}\left[C_{1}\right] /\left(\sum_{g \in C_{1}} g\right)\right\}$ (see $\left.[\mathbf{1 0}]\right)$ and that the results of [14] give an explicit description of $\mathrm{IM}_{p}^{2}$ showing that $\left|\mathrm{IM}_{p}^{2}\right|=4 p-2$. However, if $n>2$, then in [15] Heller and Reiner have shown that $\mathrm{IM}_{p}^{n}$ is infinite and, even now, there is still no explicit description of these sets.

For each natural number $b$ we also set

$$
\kappa_{n}^{b}:=\sum_{J_{1} \times \cdots \times J_{n}} \prod_{i=1}^{i=n-1} c_{J_{i}} c_{J_{i+1}},
$$

where in the sum each $J_{i}$ runs over a set of representatives of the isomorphism classes of finite abelian $p$-groups of exponent dividing $p^{i}$ and $p$-rank at most $b$ and $c_{J_{i}}$ denotes the number of conjugacy classes in Aut $\left(J_{i}\right)$ comprising elements of order dividing $p^{n}$.

We fix a primitive $p$-th root of unity $\zeta_{p}$ in $\mathbb{Q}^{c}$. In the sequel we also write $\mathrm{Cl}_{F}$ for the ideal class group of a number field $F$ and set $h_{F}:=\left|\mathrm{Cl}_{F}\right|$.

The following result is proved in Section 4.4.

Corollary 2.12. We fix a natural number $n$ and for each cyclic extension of number fields $L / K$ of degree $p^{n}$ that is unramified outside $p$ we set

$$
m_{L / K}:=\operatorname{maximum}\left\{g_{E}+\operatorname{rk}_{p}\left(\mathrm{Cl}_{E\left(\zeta_{p}\right)}\right)\right\}_{E}
$$


where in the set $E$ runs over all proper subfields of $L$ that contain $K$ and $g_{E}$ denotes the number of $p$-adic places of $E$.

For each natural number $b$ we also write $\mathcal{F}_{n}^{b}$ for the set of cyclic extensions $L / K$ of degree $p^{n}$ that are unramified outside $p$ and satisfy $m_{L / K} \leq b$.

Then the following claims are valid.

(i) For any sufficiently large $b$ the $\mathbb{Z}_{p}$-rank of $\overline{A_{L}^{p}}$ is unbounded as $L / K$ ranges over $\mathcal{F}_{n}^{b}$

(ii) For each lattice I in $\mathrm{IM}_{p}^{n}$ write $m_{I}^{b}$ for the maximal multiplicity with which I occurs as a direct summand of $\overline{A_{L}^{p}}$ for any choice of $L / K$ in $\mathcal{F}_{n}^{b}$ (where in each case $A_{L}^{p}$ is regarded as a $\mathbb{Z}_{p}\left[C_{n}\right]$-module via some choice of isomorphism of $G_{L / K}$ with $C_{n}$ ). Then one has

$$
\sum_{I \in \mathrm{IM}_{p}^{n}} m_{I}^{b} \leq p^{n(n-1) b^{2}} \cdot \kappa_{n}^{b} .
$$

Hence, only finitely many isomorphism classes of indecomposable $\mathbb{Z}_{p}\left[C_{n}\right]$-lattices occur as direct summands of any $\overline{A_{L}^{p}}$ as $L / K$ ranges over $\mathcal{F}_{n}^{b}$.

REMARK 2.13. In many cases the sets $\mathcal{F}_{n}^{b}$ contain natural Iwasawa-theoretic families of extensions $L / K$ (see Proposition 4.4). The finiteness assertion at the end of Corollary 2.12(ii) is of interest in view of the result in claim (i). This finiteness assertion naturally raises the question of whether there are general conditions under which, for given values of $n$ and $b$, one can explicitly describe the indecomposable $\mathbb{Z}_{p}\left[C_{n}\right]$-lattices that arise, up to isomorphism, as direct summands of $\overline{A_{L}^{p}}$ as $L / K$ varies over $\mathcal{F}_{n}^{b}$ ? This question is similar in spirit to that considered in a different context by Elder in $[\mathbf{1 1}$, Section 7] but appears to be difficult.

\subsection{The structure of $A_{L}^{\Sigma}$ over $p$-rational fields.}

Following Movahhedi and Nguyen Quang Do [23], a number field is said to be ' $p$ rational' if the Galois group of its maximal pro- $p$ extension that is unramified outside $p$ is a free pro- $p$ group.

It is known that a number field $K$ has this property if and only if it both validates Leopoldt's Conjecture at $p$ and is such that $A_{K}^{p}$ is torsion-free (see Jaulent and Nguyen Quang Do [17, Theorem 1.2]). We further recall that any $K$ for which both $K\left(\zeta_{p}\right)$ has a unique $p$-adic place and $h_{K\left(\zeta_{p}\right)}$ is prime to $p$ has these properties and hence in particular that, if $p$ is regular, then any absolutely abelian field of $p$-power conductor is $p$-rational (see [17, Corollary 1.3(ii)]).

As another application of our methods, in Section 5 we prove the following result.

In the sequel we write $Z(\mathcal{G})$ for the centre of a group $\mathcal{G}$.

TheOREm 2.14. Assume $K$ is p-rational, let $L$ be any finite p-power degree Galois extension of $K$ and set $G:=G_{L / K}$. Let $\Sigma$ be a finite set of places of $K$ that contains all places that are either archimedean, $p$-adic or ramify in $L / K$ and is also such that the group $A_{K}^{\Sigma}$ is torsion-free.

Then the following claims are valid. 
(i) L validates Leopoldt's Conjecture at $p$.

(ii) The $\mathbb{Q}_{p}[G]$-module spanned by $B_{L}^{\Sigma}$ is free of rank $r_{K}$. The $\mathbb{Z}_{p}[G]$-module $B_{L}^{\Sigma}$ is free if and only if one of the following conditions is satisfied:

(a) $L$ is contained in $K^{\text {cyc }}$;

(b) $G$ is cyclic, $L$ is disjoint from $K^{\text {cyc }}$ and for every proper subfield $F$ of $L$ that contains $K$ the maximal abelian extension of $F^{c y c}$ in $M_{L}^{\Sigma}$ is equal to $M_{F}^{\Sigma}$.

(iii) Fix a cyclic subgroup $C$ of $G$ and set $F:=L^{C}$.

(a) If $\gamma$ is any element of $G_{M_{L}^{\Sigma} / F}$ of infinite order that projects to a generator of $C$, then $\gamma^{|C|} \in Z\left(G_{M_{L}^{\Sigma} / F}\right) \cap A_{L}^{\Sigma}$ and the $\mathbb{Z}_{p}[C]$-module $A_{L}^{\Sigma} /\left\langle\gamma^{|C|}\right\rangle$ is free of rank $r_{F}=[G: C] \cdot r_{K}$.

(b) The $\mathbb{Z}_{p}[C]$-module $A_{L}^{\Sigma}$ is isomorphic to $\mathbb{Z}_{p}[C]^{[G: C] \cdot r_{K}} \oplus \mathbb{Z}_{p}$.

(c) If $r_{K}=0$, then $B_{L}^{\Sigma}$ vanishes. If $r_{K}>0$, then there exists an exact sequence of $\mathbb{Z}_{p}[C]$-modules

$$
0 \rightarrow \mathbb{Z}_{p}^{1-n_{C}} \rightarrow B_{L}^{\Sigma} \rightarrow \mathbb{Z}_{p}[C]^{[G: C] \cdot r_{K}} \rightarrow \mathbb{Z}_{p} /\left(\mathbb{Z}_{p} \cdot n_{C}\left[L: L \cap F^{\mathrm{cyc}}\right]\right) \rightarrow 0
$$

where $n_{C}$ is equal to 0 if $L \cap F^{\mathrm{cyc}}=F$ and equal to 1 otherwise.

REMARK 2.15. The result of Theorem 2.14(i) is not new. It has been proved both by Miki [21, Theorem 3] and by Jaulent and Nguyen Quang Do [17, Corollary 1.5] by means of arguments that are different from ours because they make critical use of Shafarevic's description [28] of the minimal number of generators and relations of the Galois group of the maximal pro- $p$ extension of $K$ unramified outside $\Sigma$. If $L / K$ is Galois of degree $p$ it has also been proved by Miki and Sato in [22] by a method closer in spirit to ours but still reliant on Sharaferevich's results. Finally note that Theorem 2.14(i) is of interest since it implies the validity of Leopoldt's Conjecture for $L$ at $p$ can be deduced from its validity for the subfield $K$.

REMARK 2.16. The modified ray class groups $A_{L}^{\Sigma} /\left\langle\gamma^{|C|}\right\rangle$ in Theorem 2.14(iii)(a) play a key role in the approach of Macias Castillo and the first author in $[\mathbf{8}]$ where they are used to reinterpret the validity of Leopoldt's Conjecture at $p$.

\subsection{Extensions of prime power conductor.}

For each natural number $n$ we now fix a primitive $p^{n}$-th root of unity $\zeta_{p^{n}}$ in $\mathbb{Q}^{c}$ with the property that $\zeta_{p^{n}}^{p}=\zeta_{p^{n-1}}$ for all $n>1$.

In this section we assume $p$ does not divide the class number of the maximal real subfield of $\mathbb{Q}\left(\zeta_{p}\right)$. (We recall the latter condition is automatically satisfied by regular primes and that Vandiver's Conjecture asserts it is satisfied by all primes.)

In this case we state several results concerning the arithmetic of abelian fields of $p$-power conductor that are obtained by specialisation of the general approach discussed above.

These include a natural analogue for ray class groups of Washington's determination of the Galois structure of ideal class groups of such fields [30, Theorem 10.14] and new 
families of explicit congruence relations between (suitably normalised) values at $s=1$ of Dirichlet $L$-series associated to characters of $p$-power conductor.

2.5.1. We first fix some more general notation.

For each natural number $n$ we set $L_{n}:=\mathbb{Q}\left(\zeta_{p^{n}}\right), G_{n}:=G_{L_{n} / \mathbb{Q}}$ and $P_{n}:=G_{L_{n} / L_{1}}$. We also write $H_{n}$ for the unique subgroup of $G_{n}$ of order $p-1$ and use the restriction map $G_{n} \rightarrow G_{1}$ to identify $H_{n}$ with $G_{1}$.

For each finite abelian group $\Gamma$ we set $\Gamma^{*}:=\operatorname{Hom}\left(\Gamma, \mathbb{Q}_{p}^{c \times}\right)$ and for each $\phi$ in $\Gamma^{*}$ we write $e_{\phi}$ for the idempotent $|\Gamma|^{-1} \sum_{\gamma \in \Gamma} \phi(\gamma) \gamma^{-1}$ of $\mathbb{Q}_{p}^{c}[\Gamma]$. We write $\mathbf{1}_{\Gamma}$ for the trivial element of $\Gamma^{*}$ and often abbreviate the idempotent $e_{\mathbf{1}_{\Gamma}}$ to $e_{\Gamma}$. For each element $x$ of $\mathbb{C}_{p}[\Gamma]$ and each $\phi$ in $\Gamma^{*}$ we write $x^{\phi}$ for the unique element of $\mathbb{C}_{p}$ that is defined by the equality $x=\sum_{\phi \in \Gamma^{*}} x^{\phi} e_{\phi}$.

We use the fact that the natural direct product decomposition $G_{n}=P_{n} \times H_{n}$ implies each element of $G_{n}^{*}$ can be written uniquely as a product $\psi \phi$ with $\psi$ in $P_{n}^{*}$ and $\phi$ in $H_{n}^{*}$ and also that for each $\phi$ in $H_{n}^{*}$ the idempotent $e_{\phi}$ belongs to $\mathbb{Z}_{p}\left[H_{n}\right]$.

We write $\tau_{n}$ for the (unique) complex conjugation in $H_{n}$ and for any $\mathbb{Z}_{p}\left[G_{n}\right]$-module $M$ we write $M^{+}$and $M^{-}$for the submodules upon which $\tau_{n}$ acts as multiplication by +1 and -1 respectively. In particular, $E^{+}$denotes the maximal real subfield of each subfield $E$ of $L_{n}$.

We also write $G_{n}^{*,-}$ and $G_{n}^{*,+}$ for the subsets of $G_{n}^{*}$ comprising characters that are odd (that is, satisfy $\chi\left(\tau_{n}\right)=-1$ ) and even (satisfy $\chi\left(\tau_{n}\right)=1$ ) respectively. We define subsets $H_{n}^{*,+}$ and $H_{n}^{*,-}$ of $H_{n}^{*}$ in a similar way and write $\omega$ for the Teichmuller character in $H_{n}^{*,-}=G_{1}^{*,-}$.

For any integer $i$ and any $\mathbb{Z}_{p}\left[G_{n}\right]$-module $M$ we write $M^{(i)}$ for the $\omega^{i}$-isotypic component $e_{\omega^{i}}(M)$ of $M$.

2.5.2. To define the necessary logarithmic resolvents and $\mathcal{L}$-invariants we fix an isomorphism of fields $j$ between $\mathbb{C}$ and the completion $\mathbb{C}_{p}$ of an algebraic closure of $\mathbb{Q}_{p}$ and, to ease notation, often suppress explicit reference to this isomorphism in what follows.

We also fix an embedding $\sigma_{n}: L_{n} \rightarrow \mathbb{Q}^{c}$, set $L_{n, p}:=\mathbb{Q}_{p} \otimes_{\mathbb{Q}} L_{n}$ (which we identify with the completion of $L_{n}$ at its unique $p$-adic place) and $\mathbb{Z}_{p} \hat{\otimes} L_{n, p}^{\times}$for the pro- $p$ completion of $L_{n, p}^{\times}$.

For any element $u$ of $\mathbb{Z}_{p} \hat{\otimes} L_{n, p}^{\times}$and any character $\chi$ in $G_{n}^{*,-}$ we then define a normalised logarithmic resolvent by setting

$$
\mathcal{L R}_{u}^{\chi}:=\frac{1}{j(2 \pi i)} \sum_{g \in G_{n}} \chi(g)^{-1} \log _{p}\left(\sigma_{n}(g(u))\right) \in \mathbb{C}_{p} .
$$

To define $\mathcal{L}$-invariants we use the composite homomorphisms of $G_{n}$-modules

$$
\log _{\infty}^{n}: \mathcal{O}_{L_{n}^{+}}^{\times} \rightarrow \prod_{\sigma} \mathbb{R}^{\times} \stackrel{(\log |\cdot|)_{\sigma}}{\longrightarrow} \prod_{\sigma} \mathbb{R}=\mathbb{R} \otimes_{\mathbb{Q}} L_{n}^{+},
$$

where $\sigma$ runs over the set of all embeddings $L_{n}^{+} \rightarrow \mathbb{Q}^{c}$ and the first arrow is the diagonal embedding, and 


$$
\log _{p}^{n}: \mathcal{O}_{L_{n}^{+}}^{\times} \rightarrow \mathcal{O}_{L_{n, p}^{+}}^{\times} \stackrel{\log _{p}}{\longrightarrow} L_{n, p}^{+}=\mathbb{Q}_{p} \otimes_{\mathbb{Q}} L_{n}^{+}
$$

where the first arrow is the obvious embedding and $\log _{p}$ denotes Iwasawa's $p$-adic logarithm.

We write $L_{n, 0}^{+}$for the kernel $\left(1-e_{G_{n}}\right) L_{n}^{+}$of the field-theoretic trace map $L_{n}^{+} \rightarrow \mathbb{Q}$. Then $\operatorname{im}\left(\log _{\infty}^{n}\right)$ is a full $\mathbb{Z}\left[G_{n}\right]$-lattice in $\mathbb{R} \otimes_{\mathbb{Q}} L_{n, 0}^{+}$and $\mathbb{Z}_{p} \cdot \operatorname{im}\left(\log _{p}^{n}\right)$ is a full $\mathbb{Z}_{p}\left[G_{n}\right]$-lattice in $\mathbb{Q}_{p} \otimes_{\mathbb{Q}} L_{n, 0}^{+}$and so for any generator $x$ of the $\mathbb{Q}\left[G_{n}\right]$-module spanned by $\mathcal{O}_{L_{n}^{+}}^{\times}$there is in $\left(\mathbb{C}_{p} \otimes_{\mathbb{Q}} L_{n, 0}^{+}\right) \oplus \mathbb{C}_{p} e_{G_{n}}$ an equality

$$
\left(j \otimes \operatorname{id}_{L_{n, 0}^{+}}\right)\left(\log _{\infty}^{n}(x)\right)+e_{G_{n}}=\mathcal{L}_{\infty, p}^{n} \cdot\left(\log _{p}^{n}(x)+e_{G_{n}}\right)
$$

for an ' $\mathcal{L}$-invariant' $\mathcal{L}_{\infty, p}^{n}$ in $\mathbb{C}_{p}\left[G_{n}\right]^{+, \times}$that is independent of the choice of $x$.

For each character $\psi$ in $G_{n}^{*}$ we usually abbreviate the scalar $\mathcal{L}_{\infty, p}^{n, \psi}$ to $\mathcal{L}_{\infty, p}^{\psi}$.

2.5.3. In order to state the main result of this section we fix a topological generator $\gamma_{\mathbb{Q}}$ of $\Gamma_{\mathbb{Q}}$. Noting $G_{n} / H_{n}$ can be identified with the quotient of $\Gamma_{\mathbb{Q}}$ of order $p^{n-1}$ we also fix a generating element $\gamma_{n}$ of $G_{n}$ that projects to the same element of $G_{n} / H_{n}$ as does $\gamma_{\mathbb{Q}}$.

We write $\chi_{\mathbb{Q}}$ for the cyclotomic character of $\Gamma_{\mathbb{Q}}$ and define an element

$$
\epsilon_{\gamma_{\mathbb{Q}}}^{n}:=\sum_{\psi \in G_{n}^{*}} \epsilon_{\gamma_{\mathbb{Q}}}^{\psi} e_{\psi}
$$

of $\mathbb{Q}_{p}\left[G_{n}\right]^{\times}$by setting for each $\psi$ in $G_{n}^{*}$

$$
\epsilon_{\gamma_{\mathbb{Q}}}^{\psi}:= \begin{cases}\log _{p}\left(\chi_{\mathbb{Q}}\left(\gamma_{\mathbb{Q}}\right)\right)^{-1}, & \text { if } \psi=\mathbf{1}_{G_{n}}, \\ \left(1-\psi\left(\gamma_{n}\right)\right)^{-1}, & \text { if } \psi\left(H_{n}\right)=1 \text { and } \psi\left(\gamma_{n}\right) \neq 1, \\ 1, & \text { if } \psi\left(H_{n}\right) \neq 1\end{cases}
$$

Finally we set

$$
\theta_{n}^{*}(1):=\left(1-p^{-1}\right) e_{\mathbf{1}_{G_{n}}}+\sum_{\psi \in G_{n}^{*} \backslash\left\{\mathbf{1}_{G_{n}}\right\}} L(\psi, 1) \cdot e_{\psi} \in \mathbb{C}_{p}\left[G_{n}\right]^{\times}
$$

(and we note that this element can be shown to be equal to the leading term at $z=1$ of the $p$-truncated equivariant Dedekind zeta function that is naturally attached to the extension $L_{n} / \mathbb{Q}$ ).

The following result will be proved in Section 6 .

THEOREM 2.17. If $p$ does not divide $h_{\mathbb{Q}\left(\zeta_{p}\right)^{+}}$, then the following claims are valid for every natural number $n$.

(i) There are isomorphisms of $\mathbb{Z}_{p}\left[G_{n}\right]$-modules

$$
A_{L_{n}}^{p} \cong B_{L_{n}}^{p} \oplus \mathbb{Z}_{p} \text { and } \overline{B_{L_{n}}^{p}} \cong \mathbb{Z}_{p}\left[G_{n}\right]^{-} .
$$


(ii) The groups $A_{L_{n} \text {, tor }}^{p}$ and $B_{L_{n}}^{p,+}$ coincide, the product $\theta_{n}^{*}(1) \cdot \epsilon_{\gamma_{Q}}^{n} \mathcal{L}_{\infty, p}^{n}$ belongs to $\mathbb{Z}_{p}\left[G_{n}\right]^{+} \cap\left(\mathbb{C}_{p}\left[G_{n}\right]^{+}\right)^{\times}$and there $i s$ an isomorphism of $\mathbb{Z}_{p}\left[G_{n}\right]$-modules

$$
A_{L_{n}, \text { tor }}^{p} \cong \mathbb{Z}_{p}\left[G_{n}\right]^{+} /\left(\theta_{n}^{*}(1) \cdot \epsilon_{\gamma_{\mathbb{Q}}}^{n} \mathcal{L}_{\infty, p}^{n}\right) .
$$

(iii) Fix an even integer $i$ with $0 \leq i \leq p-3$ and for each $\psi$ in $P_{n}^{*}$ set

$$
L^{*}\left(\psi \omega^{i}, 1\right):= \begin{cases}1-p^{-1}, & \text { if } \psi=\mathbf{1}_{P_{n}} \text { and } i=0, \\ L\left(\psi \omega^{i}, 1\right), & \text { otherwise. }\end{cases}
$$

(a) If $p$ divides the generalised Bernoulli number $B_{1, \omega^{i-1}}$, then $A_{L_{n}, \text { tor }}^{p,(i)}$ has order at least $p^{n}$ and the product $\epsilon_{\gamma_{\mathbb{Q}}}^{\omega^{i}} \cdot \mathcal{L}_{\infty, p}^{\omega^{i}} \cdot L^{*}\left(\omega^{i}, 1\right)$ belongs to $p \cdot \mathbb{Z}_{p}$.

(b) If $p$ does not divide $B_{1, \omega^{i-1}}$, then $A_{L_{n}, \text { tor }}^{p,(i)}$ is trivial and the product $\epsilon_{\gamma_{\mathbb{Q}}}^{\omega^{i}} \cdot \mathcal{L}_{\infty, p}^{\omega^{i}}$. $L^{*}\left(\omega^{i}, 1\right)$ belongs to $\mathbb{Z}_{p}^{\times}$.

(c) In all cases, for every element $g$ in $G_{n}$ the congruence

$$
\sum_{\psi \in P_{n}^{*}}\left(\psi \omega^{i}\right)(g) \cdot \epsilon_{\gamma \mathbb{Q}}^{\psi \omega^{i}} \cdot \mathcal{L}_{\infty, p}^{\psi \omega^{i}} \cdot L^{*}\left(\psi \omega^{i}, 1\right) \equiv 0\left(\bmod |G| \cdot \mathbb{Z}_{p}\right)
$$

is valid in $\mathbb{C}_{p}$.

(iv) Fix an element $u$ of $\left(\mathbb{Z}_{p} \hat{\otimes} L_{n, p}^{\times}\right)^{-}$that the global reciprocity map of $L_{n}$ sends to a generator of the (cyclic) $\mathbb{Z}_{p}\left[G_{n}\right]$-module $\overline{B_{L_{n}}^{p}}$. Then for every odd integer $i$ with $1 \leq i \leq p-2$ the product $\mathcal{L R}_{u}^{\omega^{i}} \cdot L\left(\omega^{i}, 1\right)$ belongs to $\mathbb{Z}_{p}^{\times}$and for every element $g$ of $G_{n}$ the congruence

$$
\sum_{\psi \in P_{n}^{*}}\left(\psi \omega^{i}\right)(g) \cdot \mathcal{L} \mathcal{R}_{u}^{\psi \omega^{i}} \cdot L\left(\psi \omega^{i}, 1\right) \equiv 0\left(\bmod |G| \cdot \mathbb{Z}_{p}\right)
$$

is valid in $\mathbb{C}_{p}$.

REMARK 2.18. The known validity in this case of the $p$-adic Stark conjecture at $z=1$ (as discussed by Tate in [29, Chapter VI, Section 5] where it is attributed to Serre [27]) implies that each of the expressions $\mathcal{L}_{\infty, p}^{\psi \omega^{i}} \cdot L^{*}\left(\psi \omega^{i}, 1\right)$ in Theorem 2.17(iii) can be replaced by the leading term at $z=1$ of the $p$-adic $L$-function of $\psi \omega^{i}$. A similar change can be made to the term $\theta_{n}^{*}(1) \cdot \mathcal{L}_{\infty, p}^{n}$ that occurs in the displayed isomorphism in Theorem 2.17(ii) and shows that this isomorphism gives a strong refinement of the main results of Oriat in [25]. However we have preferred not to state Theorem 2.17 in this way in order to stress the similarity between the results obtained for odd and even characters.

\section{The proofs of Theorem 2.2 and Corollary 2.4.}

\subsection{Compactly supported étale cohomology.}

In this section we recall basic properties of the complex $R \Gamma_{c, \text { ét }}\left(\mathcal{O}_{L, \Sigma}, \mathbb{Z}_{p}(1)\right)$ introduced in Section 2.2.2 and make some preliminary observations concerning connections 
between Leopoldt's Conjecture and groups of the form $B_{L}^{\Sigma}$.

We use the notation and hypotheses of Theorem 2.2. We also write $\Sigma_{L}$ for the set of all complex embeddings $L \rightarrow \mathbb{C}$ and consider the direct sum $\bigoplus_{\Sigma_{L}} \mathbb{C}$ as a $G_{L / K} \times G_{\mathbb{C} / \mathbb{R}^{-}}$ module where $G_{L / K}$ acts on $\Sigma_{L}$ via pre-composition and $G_{\mathbb{C} / \mathbb{R}}$ acts both on $\mathbb{C}$ naturally and on $\Sigma_{L}$ via post-composition.

We write $W_{L}$ for the $G_{L / K}$-submodule of $\bigoplus_{\Sigma_{L}} 2 \pi i \cdot \mathbb{Z} \subset \bigoplus_{\Sigma_{L}} \mathbb{C}$ comprising elements that are invariant under the action of $G_{\mathbb{C} / \mathbb{R}}$.

In each degree $i$ and for each ring extension $\Lambda$ of $\mathbb{Z}_{p}$ we set $H_{c, \text { ét }}^{i}\left(\mathcal{O}_{L, \Sigma}, \Lambda(1)\right):=$ $\Lambda \otimes_{\mathbb{Z}_{p}} H^{i}\left(R \Gamma_{c, \text { ét }}\left(\mathcal{O}_{L, \Sigma}, \mathbb{Z}_{p}(1)\right)\right)$.

Proposition 3.1. Let $L / K$ be a finite Galois extension of number fields and set $G:=G_{L / K}$. Let $\Sigma$ be a finite set of places of $K$ containing all places that are either archimedean, $p$-adic or ramify in $L / K$.

(i) $R \Gamma_{c, \text { ét }}\left(\mathcal{O}_{L, \Sigma}, \mathbb{Z}_{p}(1)\right)$ is an object of the subcategory $D^{\text {perf }}\left(\mathbb{Z}_{p}[G]\right)$ of $D\left(\mathbb{Z}_{p}[G]\right)$.

(ii) The Euler characteristic of $R \Gamma_{c \text {,ét }}\left(\mathcal{O}_{L, \Sigma}, \mathbb{Z}_{p}(1)\right)$ in $K_{0}\left(\mathbb{Z}_{p}[G]\right)$ vanishes.

(iii) $R \Gamma_{c \text {,ét }}\left(\mathcal{O}_{L, \Sigma}, \mathbb{Z}_{p}(1)\right)$ is acyclic outside degrees one, two and three. There are natural identifications $H_{c, \text { ét }}^{2}\left(\mathcal{O}_{L, \Sigma}, \mathbb{Z}_{p}(1)\right)=A_{L}^{\Sigma}$ and $H_{c, \text { ét }}^{3}\left(\mathcal{O}_{L, \Sigma}, \mathbb{Z}_{p}(1)\right)=\mathbb{Z}_{p}$ and a natural exact sequence

$$
0 \rightarrow \mathbb{Z}_{p} \otimes_{\mathbb{Z}} W_{L} \rightarrow H_{c, \text { ét }}^{1}\left(\mathcal{O}_{L, \Sigma}, \mathbb{Z}_{p}(1)\right) \rightarrow \mathbb{Z}_{p} \otimes_{\mathbb{Z}} \mathcal{O}_{L}^{\times} \stackrel{\lambda_{L, p}}{\longrightarrow} \bigoplus_{w} \mathbb{Z}_{p} \hat{\otimes} L_{w}^{\times}
$$

where in the direct sum $w$ runs over all $p$-adic places of $L$ and $\lambda_{L, p}$ denotes the natural diagonal localisation map.

(iv) The following conditions are equivalent.

(a) Leopoldt's Conjecture is valid for $L$ at $p$.

(b) $H_{c \text {,ét }}^{1}\left(\mathcal{O}_{L, \Sigma}, \mathbb{Z}_{p}(1)\right)$ identifies with $\mathbb{Z}_{p} \otimes_{\mathbb{Z}} W_{L}$.

(c) The $\mathbb{Q}_{p}[G]$-modules $\mathbb{Q}_{p} \cdot B_{L}^{\Sigma}$ and $\mathbb{Q}_{p} \otimes_{\mathbb{Z}} W_{L}$ are isomorphic.

Proof. These results are essentially well-known.

Claims (i) and (ii) follow from standard properties of étale cohomology (see [12, Theorem 5.1] and [7, Lemma 7] respectively).

The explicit descriptions in claim (iii) are obtained, for example, in the course of the proof of [9, Proposition 2.1].

The equivalence of conditions (a) and (b) in claim (iv) follows directly from the displayed exact sequence in claim (iii) and the fact Leopoldt's Conjecture for $L$ at $p$ is equivalent to the vanishing of $\operatorname{ker}\left(\lambda_{L, p}\right)$.

The equivalence of condition (b) and (c) relies on the fact that $\mathbb{Q}_{p}[G]$ is a semisimple $\mathbb{Q}_{p}$-algebra. In particular, whilst the exact sequence (1) implies the $\mathbb{Q}_{p}[G]$-modules $\mathbb{Q}_{p} \cdot A_{L}^{\Sigma}$ and $\left(\mathbb{Q}_{p} \cdot B_{L}^{\Sigma}\right) \oplus \mathbb{Q}_{p}$ are isomorphic, claim (ii) combines with the explicit descriptions in claim (iii) to imply $\mathbb{Q}_{p} \cdot A_{L}^{\Sigma}$ is also isomorphic to $H_{c, \text { ét }}^{1}\left(\mathcal{O}_{L, \Sigma}, \mathbb{Q}_{p}(1)\right) \oplus \mathbb{Q}_{p}$, and hence that $\mathbb{Q}_{p} \cdot B_{L}^{\Sigma}$ and $H_{c, \text { ét }}^{1}\left(\mathcal{O}_{L, \Sigma}, \mathbb{Q}_{p}(1)\right)$ are isomorphic $\mathbb{Q}_{p}[G]$-modules. 
This makes clear the implication from (b) to (c) and also implies the converse since $\operatorname{ker}\left(\lambda_{L, p}\right)$ is $\mathbb{Z}_{p}$-free and so the exact sequence in claim (iii) implies condition (b) is satisfied if and only if $H_{c \text {,ét }}^{1}\left(\mathcal{O}_{L, \Sigma}, \mathbb{Q}_{p}(1)\right)$ and $\mathbb{Q}_{p} \otimes_{\mathbb{Z}} W_{L}$ are isomorphic $\mathbb{Q}_{p}[G]$-modules.

Corollary 3.2. Let $L / K, \Sigma$ and $G$ be as in Proposition 3.1. If no archimedean place of $K$ ramifies in $L$, then the following conditions are equivalent.

(i) Leopoldt's Conjecture is valid for $L$ at $p$.

(ii) Leopoldt's Conjecture is valid for $K$ at $p$ and $B_{L}^{\Sigma}$ spans a free $\mathbb{Q}_{p}[G]$-module.

(iii) $B_{L}^{\Sigma}$ spans a free $\mathbb{Q}_{p}[G]$-module of rank $r_{K}$.

Proof. If no archimedean place of $K$ ramifies in $L$, then $W_{L}$ is a free $G$-module of rank $\mathrm{rk}_{\mathbb{Z}_{p}}\left(\mathbb{Z}_{p} \otimes_{\mathbb{Z}} W_{K}\right)=r_{K}$, one has $r_{L}=|G| \cdot r_{K}$ and Leopoldt's Conjecture for $L$ at $p$ is valid if and only if $\operatorname{dim}_{\mathbb{Q}_{p}}\left(\mathbb{Q}_{p} \cdot B_{L}^{\Sigma}\right)=|G| \cdot r_{K}$.

In particular, since the validity of Leopoldt's Conjecture is inherited by subfields the implication from (i) to (ii) follows directly from Proposition 3.1(iv).

Next we note that, under the conditions of claim (ii), one has $\operatorname{dim}_{\mathbb{Q}_{p}}\left(\mathbb{Q}_{p} \cdot B_{K}^{\Sigma}\right)=r_{K}$ and $\operatorname{dim}_{\mathbb{Q}_{p}}\left(\mathbb{Q}_{p} \cdot B_{L}^{\Sigma}\right)=|G| \cdot \operatorname{dim}_{\mathbb{Q}_{p}}\left(H_{0}\left(G, \mathbb{Q}_{p} \cdot B_{L}^{\Sigma}\right)\right)$. To deduce claim (iii) it is thus enough to show $\operatorname{dim}_{\mathbb{Q}_{p}}\left(H_{0}\left(G, \mathbb{Q}_{p} \cdot B_{L}^{\Sigma}\right)\right)=\operatorname{dim}_{\mathbb{Q}_{p}}\left(\mathbb{Q}_{p} \cdot B_{K}^{\Sigma}\right)$ and this follows immediately from Lemma 3.3(i) below.

Finally we note that claim (iii) implies $\operatorname{dim}_{\mathbb{Q}_{p}}\left(\mathbb{Q}_{p} \cdot B_{L}^{\Sigma}\right)=|G| \cdot r_{K}=r_{L}$ and hence that claim (i) is true.

Lemma 3.3. Let $L / K, \Sigma$ and $G$ be as in Proposition 3.1.

(i) Then the spaces $\mathbb{Q}_{p} \cdot H_{0}\left(G, A_{L}^{\Sigma}\right)$ and $\mathbb{Q}_{p} \cdot H_{0}\left(G, B_{L}^{\Sigma}\right)$ identify with $\mathbb{Q}_{p} \cdot A_{K}^{\Sigma}$ and $\mathbb{Q}_{p} \cdot B_{K}^{\Sigma}$ respectively.

(ii) If $G$ is cyclic of p-power order, then $H_{0}\left(G, A_{L}^{\Sigma}\right)$ identifies with $G_{M_{K}^{\Sigma} / L}$.

(iii) If $L$ is contained in $K^{\mathrm{cyc}}$, then the canonical exact sequence (1) splits and $H_{0}\left(G, B_{L}^{\Sigma}\right)$ identifies with $B_{K}^{\Sigma}$.

Proof. In view of Proposition 3.1(iii), claim (i) for the group $A_{L}^{\Sigma}$ asserts that $H_{0}\left(G, H_{c \text {,ét }}^{2}\left(\mathcal{O}_{L, \Sigma}, \mathbb{Q}_{p}(1)\right)\right)$ identifies with $H_{c \text {,ét }}^{2}\left(\mathcal{O}_{K, \Sigma}, \mathbb{Q}_{p}(1)\right)$. This identification is wellknown and is obtained, for example, by combining the hyper-tor spectral sequence with the canonical descent isomorphism $\mathbb{Z}_{p} \otimes_{\mathbb{Z}_{p}[G]}^{\mathbb{L}} R \Gamma_{c \text {,ét }}\left(\mathcal{O}_{L, \Sigma}, \mathbb{Z}_{p}(1)\right) \cong R \Gamma_{c \text {,ét }}\left(\mathcal{O}_{K, \Sigma}, \mathbb{Z}_{p}(1)\right)$ in étale cohomology.

To deduce the analogous result for $B_{L}^{\Sigma}$ one need only note that the short exact sequence of $\mathbb{Q}_{p}[G]$-modules induced by (1) splits.

If $G$ is cyclic, then Galois theory identifies $H_{0}\left(G, A_{L}^{\Sigma}\right)$ with $G_{E / L}$ where $E$ is the maximal extension of $L$ inside $M_{L}^{\Sigma}$ that is abelian over $K$. Claim (ii) is therefore true because if $G$ has $p$-power order, then $E=M_{K}^{\Sigma}$.

To prove claim (iii) we assume $L \subset K^{\mathrm{cyc}}$ and fix an element $\gamma$ of $G_{M_{L}^{\Sigma} / K}$ that projects to give a topological generator of $\Gamma_{K}$. 
Then $\gamma^{|G|}$ belongs to $A_{L}^{\Sigma}$, is central in $G_{M_{L}^{\Sigma} / K}$ and projects to give a topological generator $\gamma^{\prime}$ of $\Gamma_{L}=\Gamma_{K}^{|G|}$. The unique homomorphism of $\mathbb{Z}_{p}$-modules $\Gamma_{L} \rightarrow A_{L}^{\Sigma}$ that sends $\gamma^{\prime}$ to $\gamma^{|G|}$ is therefore a homomorphism of $\mathbb{Z}_{p}[G]$-modules that splits the extension (1).

Since this sequence splits, the result in claim (ii) identifies $H_{0}\left(G, B_{L}^{\Sigma}\right)$ with the kernel $G_{M_{K}^{\Sigma} / L^{\text {cyc }}}$ of the restriction map $G_{M_{K}^{\Sigma} / L} \rightarrow \Gamma_{L}$ and this is equal to $B_{K}^{\Sigma}$ since $K^{\text {cyc }}=L^{\text {cyc }}$. This completes the proof of claim (iii)

\subsection{The proof of Theorem 2.2.}

The key to our proof of Theorem 2.2 is provided by the following result of Macias Castillo and the first author (from $[\mathbf{8}$, Section 5]).

LEMMA 3.4. Leopoldt's Conjecture is valid at $p$ if and only if for every Galois extension $L / K$ and set of places $\Sigma$ as in the statement of Theorem 2.2 , the $G_{L / K}$-module $H_{c, \text { ét }}^{1}\left(\mathcal{O}_{L, \Sigma}, \mathbb{Z}_{p}(1)\right)$ is cohomologically-trivial.

Given this result, Theorem 2.2 follows directly from the following observation.

Proposition 3.5. Fix a finite Galois extension $L / K$ and set of places $\Sigma$ as in the statement of Theorem 2.2 and set $G:=G_{L / K}$.

(i) The following conditions are equivalent.

(a) The G-module $H_{c \text {,ét }}^{1}\left(\mathcal{O}_{L, \Sigma}, \mathbb{Z}_{p}(1)\right)$ is cohomologically-trivial.

(b) The complex $\tau^{\geq 2} R \Gamma_{c, \text { ét }}\left(\mathcal{O}_{L, \Sigma}, \mathbb{Z}_{p}(1)\right)$ belongs to $D^{\text {perf }}\left(\mathbb{Z}_{p}[G]\right)$.

(c) For each subgroup $J$ of $G$, with $E:=L^{J}$, one has both

(i) $c_{L / E}^{\Sigma, \gamma_{L}}$ generates $H^{1}\left(J, B_{L}^{\Sigma}\right)$, and

(ii) $c_{L / E}^{\Sigma, \text { ét }}$ generates $H^{2}\left(J, A_{L}^{\Sigma}\right)$ and has order $|J|$.

(ii) If L validates Leopoldt's Conjecture at p, then the conditions in claim (i) are satisfied.

Proof. We set $C^{\bullet}:=\tau^{\geq 2} R \Gamma_{c \text {,ét }}\left(\mathcal{O}_{L, \Sigma}, \mathbb{Z}_{p}(1)\right)$.

For any $\mathbb{Z}_{p}$-module $M$ and integer $m$ we also write $M[m]$ for the complex $M^{\bullet}$ with $M^{-m}=M$ and $M^{i}=0$ for all $i \neq m$ (and with all differentials the zero map).

Then, since the complex $R \Gamma_{c \text {,ét }}\left(\mathcal{O}_{L, \Sigma}, \mathbb{Z}_{p}(1)\right)$ is acyclic in degrees less than one (by Proposition 3.1(iii)), there exists a natural exact triangle in $D\left(\mathbb{Z}_{p}[G]\right)$

$$
H_{c, \text { ét }}^{1}\left(\mathcal{O}_{L, \Sigma}, \mathbb{Z}_{p}(1)\right)[-1] \rightarrow R \Gamma_{c, \text { ét }}\left(\mathcal{O}_{L, \Sigma}, \mathbb{Z}_{p}(1)\right) \rightarrow C^{\bullet} \rightarrow H_{c, \text { ét }}^{1}\left(\mathcal{O}_{L, \Sigma}, \mathbb{Z}_{p}(1)\right)[0] .
$$

In particular, since $R \Gamma_{c \text {,ét }}\left(\mathcal{O}_{L, \Sigma}, \mathbb{Z}_{p}(1)\right)$ belongs to $D^{\text {perf }}\left(\mathbb{Z}_{p}[G]\right)$ (by Proposition 3.1(i)), this triangle implies $C^{\bullet}$ belongs to $D^{\text {perf }}\left(\mathbb{Z}_{p}[G]\right)$ if and only if the complex $H_{c, \text { ét }}^{1}\left(\mathcal{O}_{L, \Sigma}, \mathbb{Z}_{p}(1)\right)[-1]$ belongs to $D^{\text {perf }}\left(\mathbb{Z}_{p}[G]\right)$.

In addition, since the $\mathbb{Z}_{p}[G]$-module $H_{c \text {,ét }}^{1}\left(\mathcal{O}_{L, \Sigma}, \mathbb{Z}_{p}(1)\right)$ is finitely generated, it is clear that $H_{c, \text { ét }}^{1}\left(\mathcal{O}_{L, \Sigma}, \mathbb{Z}_{p}(1)\right)[-1]$ belongs to $D^{\text {perf }}\left(\mathbb{Z}_{p}[G]\right)$ if and only if the $\mathbb{Z}_{p}[G]$-module $H_{c, \text { ét }}^{1}\left(\mathcal{O}_{L, \Sigma}, \mathbb{Z}_{p}(1)\right)$ has a finite projective resolution, or equivalently (by the argument of 
[3, Chapter VI, Theorem (8.12)]) that $H_{c, \text { ét }}^{1}\left(\mathcal{O}_{L, \Sigma}, \mathbb{Z}_{p}(1)\right)$ is a cohomologically-trivial $G$-module. This shows equivalence of the conditions (i)(a) and (i)(b).

We next choose, as we may, a complex $\widehat{C}^{\bullet}$ of finitely generated $\mathbb{Z}_{p}[G]$-modules that represents $C^{\bullet}$ and has the form $M \stackrel{d}{\rightarrow} N$, where $M$ occurs in degree two and $N$ is free. Such a representative gives rise to (tautological) short exact sequences of $\mathbb{Z}_{p}[G]$-modules

$$
0 \rightarrow A_{L}^{\Sigma} \rightarrow M \rightarrow \operatorname{im}(d) \rightarrow 0
$$

and

$$
0 \rightarrow \operatorname{im}(d) \rightarrow N \rightarrow \mathbb{Z}_{p} \rightarrow 0
$$

and hence, in each degree $i$ and for each subgroup $J$ of $G$, to two connecting homomorphisms in Tate cohomology

$$
\kappa_{J, 1}^{i}: \hat{H}^{i}(J, \operatorname{im}(d)) \rightarrow \hat{H}^{i+1}\left(J, A_{L}^{\Sigma}\right), \quad \kappa_{J, 2}^{i}: \hat{H}^{i}\left(J, \mathbb{Z}_{p}\right) \rightarrow \hat{H}^{i+1}(J, \operatorname{im}(d)) .
$$

Now by an argument similar to that used above (to prove equivalence of the conditions (i)(a) and (i)(b)) it is clear that the complex $\widehat{C}^{\bullet}$, and hence also $C^{\bullet}$, belongs to $D^{\text {perf }}\left(\mathbb{Z}_{p}[G]\right)$ if and only if $M$ is a cohomologically-trivial $G$-module.

In addition, by [3, Chapter VI, Theorem (8.9)], the latter condition is satisfied if and only if for every subgroup $J$ the group $\hat{H}^{i}(J, M)$ vanishes for both $i=1$ and $i=2$. By analysing the long exact cohomology sequence of (4) one finds that the latter condition is equivalent to requiring that $\kappa_{J, 1}^{0}$ is surjective, $\kappa_{J, 1}^{1}$ is bijective and $\kappa_{J, 1}^{2}$ is injective.

We next note that the group $\hat{H}^{a}\left(J, \mathbb{Z}_{p}\right)$ vanishes for $a=-1$ and $a=1$ and is isomorphic to $\mathbb{Z}_{p} /|J|$ if $a=0$. Since each homomorphism $\kappa_{J, 2}^{i}$ is bijective (as the module $N$ that occurs in (5) is a free $\mathbb{Z}_{p}[G]$-module), it follows easily that $\kappa_{J, 1}^{2}$ is injective, that $\kappa_{J, 1}^{0}$ is surjective if and only if $\hat{H}^{1}\left(J, A_{L}^{\Sigma}\right)$ vanishes and that $\kappa_{J, 1}^{1}$ is bijective if and only if $\left(\kappa_{J, 1}^{1} \circ \kappa_{J, 2}^{0}\right)\left(1_{|J|}\right)$ has order $|J|$ and generates $\hat{H}^{2}\left(J, A_{L}^{\Sigma}\right)$ where we write $1_{|J|}$ for the image of 1 in $\mathbb{Z}_{p} /|J|$.

In addition, the composite $\kappa_{J, 1}^{1} \circ \kappa_{J, 2}^{0}$ coincides, up to sign, with the map given by taking cup product with the element $c_{L / L^{J}}^{\Sigma, \text { ét }}$ of

$$
\hat{H}^{2}\left(J, A_{L}^{\Sigma}\right)=H^{2}\left(J, A_{L}^{\Sigma}\right) \cong \operatorname{YExt}_{J}^{2}\left(\mathbb{Z}_{p}, A_{L}^{\Sigma}\right)
$$

that is defined by the complex $\operatorname{res}_{\mathbb{Z}_{p}[J]}^{\mathbb{Z}_{p}[G]} C^{\bullet}$ and so one has $\left(\kappa_{J, 1}^{1} \circ \kappa_{J, 2}^{0}\right)\left(1_{|J|}\right)= \pm c_{L / L^{J}}^{\sum \text {,ét }}$. The bijectivity of $\kappa_{J, 1}^{1}$ is thus equivalent to the condition (i)(c)(ii).

To prove $C^{\bullet}$ belongs to $D^{\text {perf }}\left(\mathbb{Z}_{p}[G]\right)$ if and only if the explicit conditions in (i)(c) are satisfied it is thus enough to show that $\hat{H}^{1}\left(J, A_{L}^{\Sigma}\right)$ vanishes if and only if $c_{L^{\Sigma} / L^{J}}^{\Sigma, \gamma_{L}}$ generates $H^{1}\left(J, B_{L}^{\Sigma}\right)$. This equivalence in turn follows easily from the fact that the long exact sequence of cohomology of (1) gives an exact sequence

$$
\hat{H}^{0}\left(J, \mathbb{Z}_{p}\right) \stackrel{\alpha}{\rightarrow} H^{1}\left(J, B_{L}^{\Sigma}\right) \rightarrow H^{1}\left(J, A_{L}^{\Sigma}\right) \rightarrow H^{1}\left(J, \mathbb{Z}_{p}\right)
$$

in which $\alpha$ sends the element $1_{|J|}$ of $\hat{H}^{0}\left(J, \mathbb{Z}_{p}\right)=\mathbb{Z}_{p} /|J|$ to $c_{L / L^{J}}^{\Sigma, \gamma_{L}}$ and the group $H^{1}\left(J, \mathbb{Z}_{p}\right)$ 
vanishes.

We have proved claim (i). To prove claim (ii) we need only note that if Leopoldt's Conjecture is valid for $L$ at $p$, then Proposition 3.1(iv) implies $H_{c \text {,ét }}^{1}\left(\mathcal{O}_{L, \Sigma}, \mathbb{Z}_{p}(1)\right)$ identifies with $\mathbb{Z}_{p} \otimes_{\mathbb{Z}} W_{L}$ and so is a cohomologically-trivial $G$-module.

\subsection{The proof of Corollary 2.4.}

We again fix $L / K$ and $\Sigma$ as in Theorem 2.2 and set $G:=G_{L / K}$. To prove Corollary 2.4 we assume $L$ validates Leopoldt's Conjecture at $p$.

At the outset note the implication from (ii) to (i) is clear since if $B_{L}^{\Sigma}$ is a cohomologically-trivial $G$-module, then $H^{1}\left(G, B_{L}^{\Sigma}\right)$ vanishes and so the sequence (1) splits.

Next note that if (1) splits, then in each degree $i$ and for each $p$-subgroup $P$ of $G$ there are isomorphisms of Tate cohomology groups

$$
\hat{H}^{i-2}\left(P, \mathbb{Z}_{p}\right) \cong \hat{H}^{i}\left(P, A_{L}^{\Sigma}\right) \cong \hat{H}^{i}\left(P, B_{L}^{\Sigma}\right) \oplus \hat{H}^{i}\left(P, \mathbb{Z}_{p}\right) .
$$

Here the first map is the composite $\kappa_{P, 1}^{i-1} \circ \kappa_{P, 2}^{i-2}$ and is an isomorphism since the assumed validity of Leopoldt's Conjecture for $L$ at $p$ combines with the proof of Proposition 3.5 to imply that the maps $\kappa_{P, 1}^{i-1}$ and $\kappa_{P, 2}^{i-2}$ are both bijective.

If $i=0$, then the map (6) is an isomorphism between the abelianization $P^{\text {ab } \cong}$ $\hat{H}^{-2}\left(P, \mathbb{Z}_{p}\right)$ of $P$ and the group $\hat{H}^{0}\left(P, B_{L}^{\Sigma}\right) \oplus \mathbb{Z}_{p} /|P|$ and so induces a surjective homomorphism from $P^{\text {ab }}$ to $\mathbb{Z}_{p} /|P|$. This shows both that $P$ is cyclic and that $\hat{H}^{0}\left(P, B_{L}^{\Sigma}\right)$, and hence (since the Tate cohomology of cyclic groups is periodic of order two) also $\hat{H}^{a}\left(P, B_{L}^{\Sigma}\right)$ in every even degree $a$, vanishes.

In a similar way, the vanishing of $\hat{H}^{-1}\left(P, \mathbb{Z}_{p}\right)$ combines with (6) to imply the vanishing of $\hat{H}^{a}\left(P, B_{L}^{\Sigma}\right)$ in every odd degree $a$.

This shows that $B_{L}^{\Sigma}$ is a cohomologically-trivial $P$-module for any $p$-subgroup $P$ of $G$ and hence also a cohomologically-trivial $G$-module (by [3, Chapter VI, Proposition (8.8)]), thus verifying the implication from (i) to (ii).

It only now remains to prove the equivalence of the conditions (ii) and (iii) and to do this we fix a Sylow $p$-subgroup $P$ of $G$ and set $F:=L^{P}$.

Noting that condition (ii) implies $P$ is cyclic (by the above argument) and that the same is clearly true under condition (iii)(a) and by explicit assumption under condition (iii)(b), we assume in the rest of the argument that $P$ is cyclic.

In particular, since $B_{L}^{\Sigma}$ spans a free $\mathbb{Q}_{p}[P]$-module (by Corollary 3.2 ), in this case a Herbrand quotient argument combines with [3, Chapter VI, Proposition (8.8)] to imply $B_{L}^{\Sigma}$ is a cohomologically-trivial $G$-module if and only if for each non-trivial subgroup $C$ of $P$ the group $\hat{H}^{-1}\left(C, B_{L}^{\Sigma}\right) \cong H^{1}\left(C, B_{L}^{\Sigma}\right)$ vanishes.

In addition, if $L \subseteq F^{\text {cyc }}$, then Lemma 3.3(iii) implies (1) splits as a sequence of $\mathbb{Z}_{p}[P]$-modules and hence, by Proposition 3.5 and the assumed validity of Leopoldt's Conjecture for $L$ at $p$, that each group $H^{1}\left(C, B_{L}^{\Sigma}\right)$ vanishes.

In the sequel we will thus assume both that $P$ is cyclic and $L \not \subset F^{\text {cyc }}$ and must show, under these hypotheses, that the conditions in (iii)(b) are satisfied if and only if the $P$-module $B_{L}^{\Sigma}$ is cohomologically-trivial, or equivalently $\hat{H}^{-1}\left(C, B_{L}^{\Sigma}\right)$ vanishes for each non-trivial subgroup $C$ of $P$. 
When proving this we can also assume both $L \cap K^{\text {cyc }}=K$ and that for each subgroup $C$ as above, with $E:=L^{C}$, the maximal abelian extension of $E^{\text {cyc }}$ in $M_{L}^{\Sigma}$ is equal to $M_{E}^{\Sigma}$ since these conditions are explicitly assumed in (iii)(b) and also follow, under the present hypotheses, from the cohomological-triviality of $B_{L}^{\Sigma}$ by Lemma 3.6 below (with $L / K$ replaced by $L / F)$.

Now, as $C$ is cyclic, the last assumption implies that the restriction map $B_{L}^{\Sigma}=$ $G_{M_{L}^{\Sigma} / L^{\text {cyc }}} \rightarrow G_{M_{E}^{\Sigma} / L^{\text {cyc }}}$ induces an identification of $H_{0}\left(C, B_{L}^{\Sigma}\right)$ with $G_{M_{E}^{\Sigma} / L^{\text {cyc }}}$. In addition, the finite group $\hat{H}^{-1}\left(C, B_{L}^{\Sigma}\right)$ is equal, by its very definition, to the kernel of the $\operatorname{map} T_{C}: G_{M_{E}^{\Sigma} / L^{c y c}}=H_{0}\left(C, B_{L}^{\Sigma}\right) \rightarrow B_{L}^{\Sigma}$ induced by the action of $\sum_{c \in C} c$ on $B_{L}^{\Sigma}$ and so vanishes if and only if $T_{C}$ is injective on the torsion subgroup of $G_{M_{E}^{\Sigma} / L^{\text {cyc }}}$.

The equivalence, under the stated hypotheses, of the conditions (ii) and (iii)(b) is thus a consequence of the fact that $T_{C}$ is equal to the restriction to $G_{M_{E}^{\Sigma} / L^{\text {cyc }}}$ of the transfer map from $A_{E}^{\Sigma}=G_{M_{E}^{\Sigma} / E}=G_{M_{L}^{\Sigma} / E}^{\mathrm{ab}}$ to $G_{M_{L}^{\Sigma} / L}^{\mathrm{ab}}=G_{M_{L}^{\Sigma} / L}=A_{L}^{\Sigma}$.

This completes the proof of Corollary 2.4.

Lemma 3.6. Let $L / K$ be a cyclic p-power degree extension of number fields that is unramified outside a finite set of places $\Sigma$ of $K$ that contains all archimedean and $p$-adic places and is such that $B_{L}^{\Sigma}$ is a cohomologically-trivial $G_{L / K}$-module.

Then the following claims are valid.

(i) For any subgroup $H$ of $G_{L / L \cap K^{\text {cyc }}}$, with $F:=L^{H}$, the maximal abelian extension of $F^{\mathrm{cyc}}$ in $M_{L}^{\Sigma}$ is equal to $M_{F}^{\Sigma}$ and hence $H_{0}\left(H, B_{L}^{\Sigma}\right)$ identifies with $G_{M_{F}^{\Sigma} / L^{\mathrm{cyc}}}$.

(ii) If $L \cap K^{\mathrm{cyc}}$ validates Leopoldt's Conjecture at $p$, then either $L \subseteq K^{\mathrm{cyc}}$ or $L \cap K^{\mathrm{cyc}}=$ $K$.

Proof. With $H$ and $F$ as in claim (i) we write $E$ for the maximal abelian extension of $F^{\text {cyc }}$ in $M_{L}^{\Sigma}$.

One has $L \cap F^{\mathrm{cyc}}=F$ so the natural restriction maps give identifications $\Gamma_{L}=\Gamma_{F}$ and $G_{L^{\mathrm{cyc}} / F^{\mathrm{cyc}}}=H$.

Then, since $H$ is cyclic, the natural conjugation action of $H$ on $B_{L}^{\Sigma}$ identifies $G_{E / L^{c y c}}$ with $H_{0}\left(H, B_{L}^{\Sigma}\right)$ and so gives a field diagram of the following kind(Figure 1).

Now the stated assumption on $B_{L}^{\Sigma}$ implies the exact sequence (1) splits and hence induces an identification of $G_{E / L^{\text {cyc }}}=H_{0}\left(H, B_{L}^{\Sigma}\right)$ with the kernel of the restriction map $H_{0}\left(H, A_{L}^{\Sigma}\right) \rightarrow H_{0}\left(H, \Gamma_{L}\right)=\Gamma_{L}$. Since the restriction map $A_{L}^{\Sigma} \rightarrow A_{F}^{\Sigma}$ induces an identification of $H_{0}\left(H, A_{L}^{\Sigma}\right)$ with $G_{M_{F}^{\Sigma} / L}$ (by Lemma 3.3(ii)) this implies that $E=M_{F}^{\Sigma}$, and hence that $H_{0}\left(H, B_{L}^{\Sigma}\right)=G_{M_{F}^{\Sigma} / L^{\text {cyc }}}$, as required to prove claim (i).

To prove claim (ii) we assume $L \nsubseteq K^{\text {cyc }}$ and set $F:=L \cap K^{\text {cyc }}$ so that the subgroup $H:=G_{L / F}$ is both non-trivial and naturally isomorphic to $G_{L^{\text {cyc }} / F^{\text {cyc }}}$. Then the above argument gives a natural short exact sequence of $\mathbb{Z}_{p}[G / H]$-modules

$$
0 \rightarrow H_{0}\left(H, B_{L}^{\Sigma}\right) \rightarrow B_{F}^{\Sigma} \rightarrow G_{L^{\text {cyc }} / F^{\text {cyc }}} \rightarrow 0
$$

in which $G / H$ acts trivially on $G_{L^{\text {cyc }} / F^{\text {cyc }}}$.

Now the cohomological-triviality of $B_{L}^{\Sigma}$ as a $G$-module implies $H_{0}\left(H, B_{L}^{\Sigma}\right) \cong$ $H^{0}\left(H, B_{L}^{\Sigma}\right)$ is a cohomologically-trivial $G / H$-module. In addition, if $F$ validates 


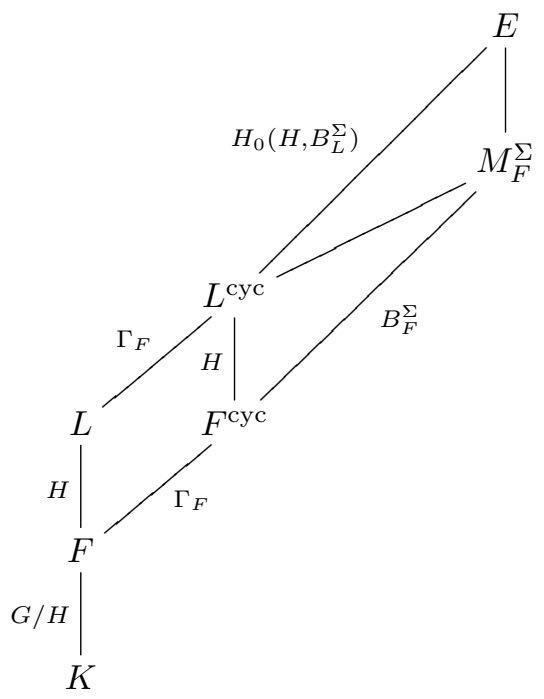

Figure 1.

Leopoldt's Conjecture at $p$, then (since $F \subseteq K^{\text {cyc }}$ ) the implication from Corollary 2.4(iii)(a) to Corollary 2.4(ii) (with $L / K$ replaced by $F / K$ ) proved above implies that $B_{F}^{\Sigma}$ is a cohomologically-trivial $G / H$-module.

From the above displayed exact sequence it therefore follows that the $G / H$-module $G_{L^{\text {cyc }} / F^{\text {cyc }}} \cong H \cong \mathbb{Z}_{p} /|H|$ is cohomologically-trivial and, as $p$ divides $|H|$, this is only possible if $G / H$ is the trivial group and hence $F=K$. This proves claim (ii).

The following result justifies Remark 2.6 and will also be useful in the sequel.

Lemma 3.7. Let $P$ be a finite abelian group of p-power order and write $\Delta_{P}$ for the number of orbits of the natural action of $G_{\mathbb{Q}_{p}^{c} / \mathbb{Q}_{p}}$ on $P^{*}:=\operatorname{Hom}\left(P, \mathbb{Q}_{p}^{c \times}\right)$. Then any nontrivial finite abelian group of p-power order that is a cohomologically-trivial P-module has order divisible by $p^{\Delta_{P}}$.

Proof. Set $R:=\mathbb{Z}_{p}[P]$. Then $R$ is a local ring and hence if $M$ is a finite abelian group of $p$-power order of the stated kind the argument of [3, Chapter VI, Theorem (8.12)] shows there exists an exact sequence of finitely generated $R$-modules of the form

$$
0 \rightarrow N \stackrel{\theta}{\rightarrow} N \rightarrow M \rightarrow 0
$$

where $N$ is free. This sequence implies that $|M|=\left|\mathbb{Z}_{p} /\left(d_{\theta}\right)\right|$ with $d_{\theta}:=\operatorname{det}_{\mathbb{Z}_{p}}(\theta)$.

To compute $d_{\theta}$ we write $r$ for the rank of $N$ and represent $\theta$ by a matrix $\Theta$ in $\mathrm{M}_{r}(R) \cap \mathrm{GL}_{r}\left(\mathbb{Q}_{p}[P]\right)$. Then $d_{\theta}=\prod_{\chi \in P^{*}} d_{\theta}(\chi)$ with $d_{\theta}(\chi):=\operatorname{det}\left(\Theta_{\chi}\right)$ where $\Theta_{\chi}$ is the matrix in $\mathrm{M}_{r}\left(\mathbb{Z}_{p}[\chi]\right)$ obtained by applying the $\mathbb{Z}_{p}$-linear extension $R \rightarrow \mathbb{Z}_{p}[\chi]$ of $\chi$ to each entry of $\Theta$. In particular, since $d_{\theta}(\omega \circ \chi)=\omega\left(d_{\theta}(\chi)\right)$ for each $\chi \in P^{*}$ and $\omega \in G_{\mathbb{Q}_{p}^{c}} / \mathbb{Q}_{p}$, one has

$$
d_{\theta}=\prod_{\chi \in \Upsilon} \mathrm{N}_{\mathbb{Q}_{p}(\chi) / \mathbb{Q}_{p}}\left(d_{\theta}(\chi)\right)
$$


where $\Upsilon$ is a set of representatives of the orbits of the action of $G_{\mathbb{Q}_{p}^{c} / \mathbb{Q}_{p}}$ on $P^{*}$.

Upon taking $P$-coinvariants of (7) we obtain an exact sequence of $\mathbb{Z}_{p}$-modules $0 \rightarrow$ $H_{0}(P, N) \stackrel{H_{0}(P, \theta)}{\longrightarrow} H_{0}(P, N) \rightarrow H_{0}(P, M) \rightarrow 0$ and, since $H_{0}(P, M)$ is non-zero, this sequence implies that $d_{\theta}\left(\mathbf{1}_{P}\right)$ belongs to the ideal $(p)$ of $\mathbb{Z}_{p}$.

In addition, if for each $\chi \in \Upsilon$ we write $\mathfrak{p}_{\chi}$ for the maximal ideal of $\mathbb{Z}_{p}[\chi]$, then one has $d_{\theta}(\chi) \equiv d_{\theta}\left(\mathbf{1}_{P}\right)$ modulo $\mathfrak{p}_{\chi}$. This implies $d_{\theta}(\chi)$ belongs to $\mathfrak{p}_{\chi}$ and hence that $\mathrm{N}_{\mathbb{Q}_{p}(\chi) / \mathbb{Q}_{p}}\left(d_{\theta}(\chi)\right)$ belongs to $(p)$.

These observations combine with (8) to imply $d_{\theta}$ belongs to $(p)^{|\Upsilon|}$ and hence that $|M|$ is divisible by $p^{|\Upsilon|}=p^{\Delta_{P}}$, as claimed.

\section{The proofs of Theorem 2.7 and Corollary 2.12 .}

\subsection{A result of Yakovlev.}

A key role in these arguments is played by a purely representation-theoretic result of Yakovlev in $[\mathbf{3 1}]$.

To recall this result we fix a cyclic group $\Gamma$ of order $p^{n}$ and for each integer $i$ with $0 \leq i \leq n$ write $\Gamma_{i}$ for the subgroup of $\Gamma$ of order $p^{i}$.

Then the results of [31, Theorem 2.4 and Lemma 5.2] combine to imply that if $M$ and $N$ are any $\mathbb{Z}_{p}[\Gamma]$-lattices for which, for each integer $i$ with $1 \leq i<n$, there exists an isomorphism of $\mathbb{Z}_{p}\left[\Gamma / \Gamma_{i}\right]$-modules $\theta_{i}: \hat{H}^{-1}\left(\Gamma_{i}, M\right) \rightarrow \hat{H}^{-1}\left(\Gamma_{i}, N\right)$ that lies in commutative diagrams
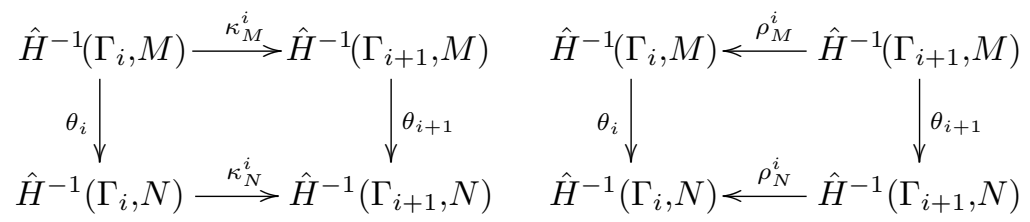

where the horizontal arrows are the natural corestriction and restriction homomorphisms, then there are isomorphisms of $\mathbb{Z}_{p}[\Gamma]$-modules of the form

$$
M \cong X \oplus \bigoplus_{i=0}^{i=n} \mathbb{Z}_{p}\left[\Gamma / \Gamma_{i}\right]^{a_{i}} \quad \text { and } \quad N \cong X \oplus \bigoplus_{i=0}^{i=n} \mathbb{Z}_{p}\left[\Gamma / \Gamma_{i}\right]^{b_{i}}
$$

for a suitable $\mathbb{Z}_{p}[\Gamma]$-lattice $X$ and suitable non-negative integers $a_{i}$ and $b_{i}$.

\subsection{The proof of Theorem 2.7.}

We use the notation and hypotheses of Theorem 2.7. We also set $A:=A_{L}^{\Sigma}$ and $G:=G_{L / K}$ and for each integer $i$ with $0 \leq i \leq n$ also $G_{i}:=G_{L / L_{i}}$ and $T_{i}:=T_{L / L_{i}}^{\Sigma}$.

We start by making a useful technical observation.

LEMMA 4.1. Fix an integer $i$ with $0 \leq i \leq n$.

(i) The group $T_{i}$ is naturally isomorphic as a $\mathbb{Z}_{p}\left[G / G_{i}\right]$-module to $\hat{H}^{-1}\left(G_{i}, \bar{A}\right)$.

(ii) If $i<n$, then the corestriction map $\hat{H}^{-1}\left(G_{i}, \bar{A}\right) \rightarrow \hat{H}^{-1}\left(G_{i+1}, \bar{A}\right)$ corresponds, under the isomorphisms in claim (i), to the homomorphism $T_{i} \rightarrow T_{i+1}$ that is 
induced by the natural restriction map $A_{L_{i}}^{\Sigma} \rightarrow A_{L_{i+1}}^{\Sigma}$.

(iii) If $i>0$, then the restriction map $\hat{H}^{-1}\left(G_{i}, \bar{A}\right) \rightarrow \hat{H}^{-1}\left(G_{i-1}, \bar{A}\right)$ corresponds, under the isomorphisms in claim (i), to the homomorphism $T_{i} \rightarrow T_{i-1}$ that is induced by the map $G_{M_{L_{i}}^{\Sigma} / L} \rightarrow G_{M_{L_{i-1}}^{\Sigma} / L}$ which sends $x$ to $\sum_{c \in G_{L_{i-1} / L_{i}}} c(\tilde{x})$ where $\tilde{x}$ is any lift of $x$ to $G_{M_{L_{i-1}}^{\Sigma} / L}$.

Proof. Upon taking $G_{i}$-coinvariants of the tautological exact sequence

$$
0 \rightarrow A_{\text {tor }} \rightarrow A \rightarrow \bar{A} \rightarrow 0,
$$

recalling that $H_{0}\left(G_{i}, A\right)$ identifies with $G_{M_{L_{i}}^{\Sigma} / L}$ (by Lemma 3.3(ii)) and then passing to torsion subgroups in the resulting exact sequence one obtains an exact sequence of $\mathbb{Z}_{p}\left[G / G_{i}\right]$-modules

$$
A_{\text {tor }} \stackrel{\pi_{L_{i}}^{L}}{\longrightarrow} G_{M_{L_{i}}^{\Sigma} / L, \text { tor }} \rightarrow H_{0}\left(G_{i}, \bar{A}\right)_{\text {tor }} \rightarrow 0
$$

Given this exact sequence and the definition of $T_{i}$ as the cokernel of $\pi_{L_{i}}^{L}$, the isomorphism in claim (i) follows from the fact that $H_{0}\left(G_{i}, \bar{A}\right)_{\text {tor }}$ is equal to $\hat{H}^{-1}\left(G_{i}, \bar{A}\right)$. Indeed, since $\hat{H}^{-1}\left(G_{i}, \bar{A}\right)$ is finite and $\bar{A}$ is $\mathbb{Z}_{p}$-free, the latter equality follows immediately from the tautological exact sequence

$$
0 \rightarrow \hat{H}^{-1}\left(G_{i}, \bar{A}\right) \stackrel{\subseteq}{\rightarrow} H_{0}\left(G_{i}, \bar{A}\right) \rightarrow H^{0}\left(G_{i}, \bar{A}\right) \rightarrow \hat{H}^{0}\left(G_{i}, \bar{A}\right) \rightarrow 0
$$

where the third arrow is induced by the action of the element $\sum_{g \in G_{i}} g$ of $\mathbb{Z}_{p}[G]$.

Given these isomorphisms, the assertions of claims (ii) and (iii) follow by straightforward computation (which we leave to the reader).

To prove Theorem 2.7 we now apply the result of Yakovlev recalled in Section 4.1 with $\Gamma=G$ and $M=\bar{A}$. In this context Lemma 4.1 implies that the upper rows of the diagrams (9) for each integer $i$ collectively correspond to the diagrams that are described in (2).

Given Yakovlev's theorem, the proof of Theorem 2.7 will therefore be complete if we show that, given an isomorphism of $\mathbb{Z}_{p}[G]$-modules of the form $\bar{A} \cong X \oplus X^{\prime}$ in which $X$ is uniquely determined up to isomorphism and $X^{\prime}$ is a module of the form $\bigoplus_{i=0}^{i=n} \mathbb{Z}_{p}\left[G / G_{i}\right]^{a_{i}}$ for suitable non-negative integers $a_{i}$, then $X^{\prime}$ is determined up to isomorphism as a $\mathbb{Z}_{p}[G]$ module by $r_{K}$ and the integers $\delta_{L_{i}}$ for each integer $i$ with $0 \leq i \leq n$.

But the explicit structure of the module $X^{\prime}$ makes it clear that it is determined up to isomorphism by the integers $\operatorname{dim}_{\mathbb{Q}_{p}}\left(\mathbb{Q}_{p} \cdot H^{0}\left(G_{i}, X^{\prime}\right)\right)$ for each integer $i$ with $0 \leq i \leq n$, and so the required fact follows from the equalities

$$
\begin{aligned}
\operatorname{dim}_{\mathbb{Q}_{p}}\left(\mathbb{Q}_{p} \cdot H^{0}\left(G_{i}, X^{\prime}\right)\right) & =\operatorname{dim}_{\mathbb{Q}_{p}}\left(\mathbb{Q}_{p} \cdot H^{0}\left(G_{i}, \bar{A}\right)\right)-\operatorname{dim}_{\mathbb{Q}_{p}}\left(\mathbb{Q}_{p} \cdot H^{0}\left(G_{i}, X\right)\right) \\
& =\operatorname{dim}_{\mathbb{Q}_{p}}\left(\mathbb{Q}_{p} \cdot A_{L_{i}}^{\Sigma}\right)-\operatorname{dim}_{\mathbb{Q}_{p}}\left(\mathbb{Q}_{p} \cdot H^{0}\left(G_{i}, X\right)\right) \\
& =r_{L_{i}}+\delta_{L_{i}}-\operatorname{dim}_{\mathbb{Q}_{p}}\left(\mathbb{Q}_{p} \cdot H^{0}\left(G_{i}, X\right)\right) \\
& =p^{n_{i}} \cdot r_{K}+\delta_{L_{i}}-\operatorname{dim}_{\mathbb{Q}_{p}}\left(\mathbb{Q}_{p} \cdot H^{0}\left(G_{i}, X\right)\right),
\end{aligned}
$$


where the second equality is true because Lemma 3.3(i) implies that the $\mathbb{Q}_{p}$-spaces $\mathbb{Q}_{p}$. $H^{0}\left(G_{i}, \bar{A}\right) \cong \mathbb{Q}_{p} \cdot H_{0}\left(G_{i}, A\right)$ and $\mathbb{Q}_{p} \cdot A_{L_{i}}^{\Sigma}$ are isomorphic.

This completes the proof of Theorem 2.7.

REMARK 4.2. If, in the notation of Section 4.1, $M$ is any lattice for which $\hat{H}^{-1}\left(\Gamma_{i}, M\right)$ vanishes for each integer $i$, then in the decomposition (10) we may take the lattice $N$, and hence also $X$, to be the zero module and therefore deduce that $M$ is itself a direct sum of modules of the form $\mathbb{Z}_{p}\left[\Gamma / \Gamma_{i}\right]$. This observation (with $\Gamma=G$ and $M=\overline{A_{L}^{\Sigma}}$ ) combines with the explicit computation of Lemma 4.1(i) to justify the first assertion of Remark 2.10.

\subsection{The vanishing of $T_{L / K}^{\Sigma}$.}

We recall that an extension of number fields $L / K$ is said to be ' $\mathbb{Z}_{p}$-extendable' if there exists a Galois extension $L^{\prime}$ of $K$ which contains $L$ and is such that $G_{L^{\prime} / K}$ is isomorphic to $\mathbb{Z}_{p}$. Such extensions have been extensively investigated in the literature, for example by Seo in $[\mathbf{2 6}]$.

It is clear that for any finite set of places $\Sigma$ of $K$ that contains all $p$-adic places the group $A_{K}^{\Sigma}$ is torsion-free if and only if all cyclic degree $p$ extensions of $K$ that are unramified outside $\Sigma$ are $\mathbb{Z}_{p}$-extendable.

In the following result we use the concept of $\mathbb{Z}_{p}$-extendability to give a useful criterion for the vanishing of the groups $T_{L / K}^{\Sigma}$ that occur in Theorem 2.7.

For a number field $E$ we write $E^{\max }$ for the maximal $\mathbb{Z}_{p}$-power extension of $E$ inside $\mathbb{Q}^{c}$.

Lemma 4.3. Assume $L / K$ is cyclic of finite p-power degree and unramified outside a finite set of places $\Sigma$ that contains all p-adic places.

(i) $T_{L / K}^{\Sigma}$ is naturally isomorphic to the torsion subgroup of $G_{\left(L^{\max } \cap M_{K}^{\Sigma}\right) / L}$.

(ii) The following conditions are equivalent:

(a) $T_{L / K}^{\Sigma}$ vanishes.

(b) For any degree $p$ extension $F$ of $L$ that is abelian over $K$ one has

$F / L$ is $\mathbb{Z}_{p}$-extendable

$\Longleftrightarrow F$ is contained in a $\mathbb{Z}_{p}$-extension of $L$ that is abelian over $K$.

(iii) If $L / K$ is $\mathbb{Z}_{p}$-extendable, then $T_{L / K}^{\Sigma}$ is equal to the cokernel of the restriction map $A_{L, \text { tor }}^{\Sigma} \rightarrow A_{K, \text { tor }}^{\Sigma}$. In this case the following conditions are equivalent:

(a) $T_{L / K}^{\Sigma}$ vanishes.

(b) $L^{\max } \cap M_{K}^{\Sigma}=K^{\max }$.

(c) For any degree $p$ Galois extension $K^{\prime}$ of $K$ in $M_{K}^{\Sigma}$ that is not $\mathbb{Z}_{p}$-extendable the extension $L K^{\prime} / L$ is not $\mathbb{Z}_{p}$-extendable. 
Proof. To prove claim (i) we note $A_{L, \text { tor }}^{\Sigma}=G_{M_{L}^{\Sigma} / L^{\max }}$ and that $G_{M_{K}^{\Sigma} / L \text {,tor }}=$ $G_{M_{K}^{\Sigma} / E}$ with $E$ a subfield of $L^{\max }$ such that $E / L$ is $\mathbb{Z}_{p}$-free and $\left(M_{K}^{\Sigma} \cap L^{\max }\right) / E$ is finite. The claimed isomorphism thus follows from the fact that the image of $G_{M_{L}^{\Sigma} / L^{\max }}$ under the restriction map $G_{M_{L}^{\Sigma} / L} \rightarrow G_{M_{K}^{\Sigma} / L}$ is $G_{M_{K}^{\Sigma} /\left(M_{K}^{\Sigma} \cap L^{\max }\right)}$.

Set $E^{*}:=M_{K}^{\Sigma} \cap L^{\max }$. Then claim (i) implies $T_{L / K}^{\Sigma}$ vanishes if and only if $G_{E^{*} / L}$ is $\mathbb{Z}_{p}$-free. The latter condition is satisfied if and only if every intermediate field of $E^{*} / L$ of degree $p$ over $L$ is contained in an intermediate field that is a $\mathbb{Z}_{p}$-extension of $L$ and hence is equivalent to the condition in claim (ii)(b).

The first assertion of claim (iii) is true because if $L / K$ is $\mathbb{Z}_{p}$-extendable, then $L \subseteq$ $K^{\max }$ and so $G_{M_{K}^{\Sigma} / L, \text { tor }}=G_{M_{K}^{\Sigma} / K^{\max }}=A_{K, \text { tor }}^{\Sigma}$.

In this case therefore the group $T_{L / K}^{\Sigma}$ vanishes if and only if the natural restriction map $\theta: A_{L, \text { tor }}^{\Sigma} \rightarrow A_{K, \text { tor }}^{\Sigma}$ is surjective, or equivalently $L^{\max } \cap M_{K}^{\Sigma}=K^{\max }$.

If the latter equality is valid, then for any degree $p$ Galois extension $K^{\prime}$ of $K$ in $M_{K}^{\Sigma}$ with $K^{\prime} \not \subset K^{\max }$ one has $L K^{\prime} \not \subset L^{\max }$ (since $\left.L K^{\prime} \subset M_{K}^{\Sigma}\right)$.

To prove the converse implication, and hence complete the proof of claim (iii), we argue by contradiction and thus assume $\operatorname{im}(\theta) \neq A_{K, \text { tor }}^{\Sigma}$. After choosing a direct sum

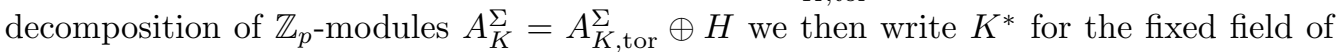
$M_{K}^{\Sigma}$ with respect to the subgroup $\operatorname{im}(\theta) \oplus H$. Then $K^{*} \neq K$ and $G_{K^{*} / K}$ is naturally isomorphic to $A_{K, \text { tor }}^{\Sigma} / \operatorname{im}(\theta)$. Hence, for any intermediate field $K^{\prime}$ of $K^{*} / K$ with $K^{\prime} \neq K$ one has $K^{\prime} \not \subset K^{\max }$ and $L K^{\prime} \subset M_{K}^{\Sigma} \cap L^{\max }$ and this contradicts the condition in (iii) (c).

\subsection{The proof of Corollary 2.12.}

We continue for the moment to use the notation of Section 4.1.

4.4.1. For any natural number $d$ and each indecomposable lattice $I$ in $\operatorname{IM}_{p}^{n}$ we write $\widetilde{m}_{I}^{d}$ for the maximal multiplicity with which $I$ occurs as a direct summand of any $\mathbb{Z}_{p}[\Gamma]$-lattice $N$ for which one has $\operatorname{rk}_{p}\left(\hat{H}^{-1}\left(\Gamma_{i}, N\right)\right) \leq d$ for all $i$ with $1 \leq i \leq n$.

Then the key point in the proof of Corollary 2.12(ii) is that Yakovlev's theorem combines with an analysis of the diagrams (9) to show that

$$
\sum_{I \in \mathrm{IM}_{p}^{n}} \widetilde{m}_{I}^{d} \leq p^{n(n-1) d^{2}} \cdot \kappa_{n}^{d}
$$

where $\kappa_{n}^{d}$ is the integer defined just prior to the statement of Corollary 2.12 (for a proof of this fact see [6, Lemma 3.2]).

To deduce the inequality (3) from this it is thus enough to note that if for any non-trivial subgroup $H$ of $G_{L / K}$ we set $E:=L^{H}$, then one has

$$
\begin{aligned}
\operatorname{rk}_{p}\left(\hat{H}^{-1}\left(H, \overline{A_{L}^{p}}\right)\right)=\operatorname{rk}_{p}\left(T_{L / E}^{p}\right) \leq \operatorname{rk}_{p}\left(A_{E, \text { tor }}^{p}\right) & \\
& =g_{E}-\nu_{E}+\operatorname{rk}_{p}\left(\mathrm{Cl}_{E\left(\zeta_{p}\right)}^{\prime}\right)-\delta_{E} \leq m_{L / K},
\end{aligned}
$$

where $g_{E}$ denotes the number of $p$-adic places of $E, \nu_{E}$ is either 0 or 1 (depending on whether $E$ contains $\zeta_{p}$ or not) and $\mathrm{Cl}_{E\left(\zeta_{p}\right)}^{\prime}$ is a subquotient of $\mathrm{Cl}_{E\left(\zeta_{p}\right)}$. Here the first equality follows from Lemma 4.1(i) and the second is proved by Gras in [13, Theorem I2], 
the first inequality is true since $T_{L / E}^{p}$ is (by its definition) a subquotient of $A_{E \text {,tor }}^{p}$ and the second inequality follows directly from the definition of $m_{L / K}$ in the statement of Corollary 2.12 and the fact that each Leopoldt defect $\delta_{E}$ is non-negative.

To complete the proof of Corollary 2.12(ii) it is now enough to note that the inequality (3) directly implies that $m_{I}^{b}$ can be non-zero for only finitely many lattices $I$ in $\mathrm{IM}_{p}^{n}$.

4.4.2. The following result justifies the first sentence of Remark 2.13 and is also key to the proof of Corollary 2.12(i).

For any subfield $F$ of $\mathbb{Q}^{c}$ we write $X_{F}$ for the Galois group of the maximal unramified abelian pro- $p$ extension of $F$.

Proposition 4.4. Let $E_{\infty}$ be a $\mathbb{Z}_{p}$-extension of a number field $E$ for which all $p$ adic places of $E$ have open decomposition groups in $G_{E_{\infty} / E}$ and the $\mathbb{Z}_{p}$-module $X_{E_{\infty}\left(\zeta_{p}\right)}$ is finitely generated. Let $F$ be a finite p-power degree extension of $E_{\infty}$ that is both unramified outside $p$ and Galois over E.

Then there exists an integer $d$ that depends only upon $F / E$ and is such that $g_{L}+$ $\operatorname{rk}_{p}\left(\mathrm{Cl}_{L\left(\zeta_{p}\right)}\right) \leq d$ for all finite extensions $L$ of $E$ in $F$.

Proof. The stated assumption on the decomposition behaviour of $p$-adic places in $F / E$ implies $g_{L}$ is bounded above (independently of $L$ ) as $L$ varies over finite extensions of $E$ in $F$ and so it suffices to show the same is true of $\operatorname{rk}_{p}\left(\mathrm{Cl}_{L\left(\zeta_{p}\right)}\right)$.

For any $L$ as above the compositum of $L\left(\zeta_{p}\right)$ and $E_{\infty}$ is an intermediate field of $F\left(\zeta_{p}\right) / E_{\infty}\left(\zeta_{p}\right)$ that is a $\mathbb{Z}_{p}$-extension of $L\left(\zeta_{p}\right)$. Since there are only finitely many intermediate fields of $F\left(\zeta_{p}\right) / E_{\infty}\left(\zeta_{p}\right)$ a standard Iwasawa-theoretic argument (as in [30, Proposition 13.23]) means it will be enough for us to show that, for each such intermediate field $D$, the $\mathbb{Z}_{p}$-module $X_{D}$ is finitely generated.

To show this we note each such $D$ is a finite $p$-power degree extension of $E_{\infty}\left(\zeta_{p}\right)$ and hence that one can choose a finite chain of subgroups

$$
G_{F\left(\zeta_{p}\right) / D}=J_{0} \unlhd J_{1} \unlhd \cdots \unlhd J_{m}=G_{F\left(\zeta_{p}\right) / E_{\infty}\left(\zeta_{p}\right)}
$$

with $\left|J_{i+1} / J_{i}\right|=p$ for all $i$ with $0 \leq i<m$. For each such $i$ we set $D_{i}:=F\left(\zeta_{p}\right)^{J_{i}}$. Then $D_{0}=D, D_{m}=E_{\infty}\left(\zeta_{p}\right)$ and each extension $D_{i} / D_{i+1}$ is Galois of degree $p$.

Now the stated assumptions imply each field $D_{i}$ has only finitely many $p$-adic places and $X_{D_{m}}=X_{E_{\infty}\left(\zeta_{p}\right)}$ is a finitely generated $\mathbb{Z}_{p}$-module. The required result can therefore be obtained by successively applying the result of Lemma 4.5 below to each of the extensions $D_{i} / D_{i+1}$ starting at $i=m-1$ and descending to $i=0$.

LEMma 4.5. Let $D$ be a subfield of $\mathbb{Q}^{c}$ with only finitely many p-adic places and such that the $\mathbb{Z}_{p}$-module $X_{D}$ is finitely generated. Then for any Galois extension $D^{\prime}$ of $D$ of degree $p$ the $\mathbb{Z}_{p}$-module $X_{D^{\prime}}$ is finitely generated.

Proof. Write $M_{D^{\prime}}$ and $M_{D}$ for the maximal unramified abelian pro- $p$ extensions of $D^{\prime}$ and $D$ and set $\Delta:=G_{D^{\prime} / D}$.

Then $M_{D^{\prime}} / D$ is Galois and $H_{0}\left(\Delta, X_{D^{\prime}}\right)$ identifies with $G_{M / D^{\prime}}$ where $M$ is the maximal abelian extension of $D$ in $M_{D^{\prime}}$. 
The inertia degree in $M / D$ of each of the finitely many $p$-adic places of $D$ is at most $p$ and so the subgroup $I$ of $G_{M / D}$ that is generated by the inertia subgroups of these places is finite. In addition, the fixed field $M^{I}$ is a subfield of $M_{D}$ and so $\left(G_{M / D}\right) / I \cong G_{M^{I} / D}$ is isomorphic to a quotient of the finitely generated $\mathbb{Z}_{p^{-}}$-module $X_{D}$. Since $I$ is finite, this

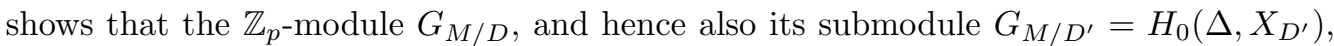
is finitely generated.

Then, since $\Delta$ is finite, Nakayama's Lemma implies that the $\mathbb{Z}_{p}$-module $X_{D^{\prime}}$ is finitely generated, as required.

To prove Corollary 2.12(i) we fix a complex absolutely abelian field $E$ and recall that the $\mathbb{Z}_{p}$-module $X_{E^{\text {cyc }}\left(\zeta_{p}\right)}$ is known to be finitely generated (this is equivalent to the vanishing of Iwasawa's $\mu$-invariant for the $\mathbb{Z}_{p}$-extension $\left.E^{\text {cyc }}\left(\zeta_{p}\right) / E\left(\zeta_{p}\right)\right)$.

From Proposition 4.4 (with $F=E_{\infty}=E^{\text {cyc }}$ ) there is therefore an integer $d$ such that $g_{E_{m}}+\operatorname{rk}_{p}\left(\mathrm{Cl}_{E_{m}\left(\zeta_{p}\right)}\right) \leq d$ for all natural numbers $m$, where $E_{m}$ denotes the unique intermediate field of $E^{\text {cyc }} / E$ of degree $p^{m}$ over $E$.

In particular, if $b$ is any integer with $b \geq d$, then for any natural numbers $n$ and $a$ the extension $E_{a+n} / E_{a}$ belongs to $\mathcal{F}_{n}^{b}$.

It is thus sufficient to note that, since each field $E_{a+n}$ is both totally complex and validates Leopoldt's Conjecture at $p$ (by Brumer [4]), Corollary 3.2 implies that the $\mathbb{Z}_{p}$-rank of $\overline{A_{E_{a+n}}^{p}}$ is $p^{a+n} \cdot r_{E}+1=\left(p^{a+n}[E: \mathbb{Q}]\right) / 2+1$.

This completes the proof of Corollary 2.12.

\section{The proof of Theorem 2.14 .}

We start the proof by making an important technical observation.

Proposition 5.1. Let $L / K$ be a cyclic extension of number fields of degree $p^{n}$ and $\Sigma$ a finite set of places of $K$ containing all places that are either archimedean, $p$-adic or ramify in $L / K$. Set $G:=G_{L / K}$ and fix an element $\gamma$ of $G_{M_{L}^{\Sigma} / K}$ that has infinite order and projects to give a generator of $G$. For each integer $i$ with $0 \leq i \leq n$ write $L_{i}$ for the intermediate field of $L / K$ that has degree $p^{i}$ over $K$ and $\gamma_{i}$ for the image of $\gamma^{p^{i}}$ in $A_{L_{i}}^{\Sigma}$.

If both $K$ validates Leopoldt's Conjecture at $p$ and the group $A_{K}^{\Sigma}$ is torsion-free, then $L$ validates Leopoldt's Conjecture at $p$ and for each integer $i$ the groups $A_{L_{i}}^{\Sigma}$ and $A_{L_{i}}^{\Sigma} /\left\langle\gamma_{i}\right\rangle$ are torsion-free.

Proof. For integers $i$ and $j$ with $0 \leq i<j \leq n$ we set $A_{i}:=A_{L_{i}}^{\Sigma}, D_{i}:=A_{L_{i}}^{\Sigma} /\left\langle\gamma_{i}\right\rangle$ and $Q_{j, i}:=G_{L_{j} / L_{i}}$.

For each integer $i$ as above one can use the multiplication-by- $p$ map on the tautological exact sequence $0 \rightarrow\left\langle\gamma_{i}\right\rangle \rightarrow A_{i} \rightarrow D_{i} \rightarrow 0$ to obtain an exact sequence

$$
0 \rightarrow A_{i,[p]} \rightarrow D_{i,[p]} \rightarrow\left\langle\gamma_{i}\right\rangle /\left\langle\gamma_{i}^{p}\right\rangle \stackrel{d_{i}}{\rightarrow} A_{i} /\left(A_{i}\right)^{p}
$$

Since the image of $\gamma_{i}$ generates $G_{L_{i+1} / L_{i}}$ one has $\gamma_{i} \notin\left(A_{i}\right)^{p}$. The map $d_{i}$ is thus injective and so the above sequence implies that the natural map $A_{i,[p]} \rightarrow D_{i,[p]}$ is bijective. This implies, in particular, that the group $D_{i}$ is torsion-free if and only if the group $A_{i}$ is torsion-free. 
Until further notice, we now assume that the integer $i$ is such that the following hypothesis is satisfied.

$(*)_{i} L_{i}$ validates Leopoldt's Conjecture at $p$ and the group $D_{i}=A_{i} /\left\langle\gamma_{i}\right\rangle$ is torsion-free.

In this case, for each integer $j$ with $i<j \leq n$ the group $H_{0}\left(Q_{j, i}, A_{j}\right)$ identifies with a subgroup of $A_{i}$ and the torsion subgroup of $H_{0}\left(Q_{j, i}, D_{j}\right)=H_{0}\left(Q_{j, i}, A_{j}\right) /\left\langle\gamma_{i}^{p^{j-i}}\right\rangle$ is equal to

$$
\left(A_{i} /\left\langle\gamma_{i}^{p^{j-i}}\right\rangle\right)_{\text {tor }} \cap H_{0}\left(Q_{j, i}, A_{j}\right) /\left\langle\gamma_{i}^{p^{j-i}}\right\rangle=\left\langle\gamma_{i}\right\rangle /\left\langle\gamma_{i}^{p^{j-i}}\right\rangle \cap H_{0}\left(Q_{j, i}, A_{j}\right) /\left\langle\gamma_{i}^{p^{j-i}}\right\rangle .
$$

Furthermore, this group vanishes since for any integer $a$ with $0 \leq a<j-i$ the element $\gamma_{i}^{p^{a}}$ does not act trivially on $L_{j}$ and so does not lie in $H_{0}\left(Q_{j, i}, \overline{A_{j}}\right)$.

It follows that under hypothesis $(*)_{i}$ the group $H_{0}\left(Q_{j, i}, D_{j}\right)$ is torsion-free and hence, by the same argument as used in the proof of Lemma 4.1 , the group $\hat{H}^{-1}\left(Q_{j, i}, \overline{D_{j}}\right)$ vanishes.

In the case $j=i+1$ we set $Q_{i}:=Q_{j, i}$. Then, since $Q_{i}$ has order $p$, there are only three isomorphism classes of indecomposable $\mathbb{Z}_{p}\left[Q_{i}\right]$-lattices, represented by $\mathbb{Z}_{p}, \mathbb{Z}_{p}\left[Q_{i}\right]$ and $\mathbb{Z}_{p}\left[Q_{i}\right] /\left(\sum_{g \in Q_{i}} g\right)$, each being endowed with the natural $Q_{i}$-action (cf. the discussion before Corollary 2.12). One also knows that the groups $\hat{H}^{-1}\left(Q_{i}, \mathbb{Z}_{p}\right)$ and $\hat{H}^{-1}\left(Q_{i}, \mathbb{Z}_{p}\left[Q_{i}\right]\right)$ vanish and that $\hat{H}^{-1}\left(Q_{i}, \mathbb{Z}_{p}\left[Q_{i}\right] /\left(\sum_{g \in Q_{i}} g\right)\right)$ has order $p$.

Thus, since $\hat{H}^{-1}\left(Q_{i}, \overline{D_{i+1}}\right)$ vanishes, the Krull-Schmidt theorem implies that the $\mathbb{Z}_{p}\left[Q_{i}\right]$-module $\overline{D_{i+1}}$ is isomorphic to a direct sum of the form $\mathbb{Z}_{p}^{a} \oplus \mathbb{Z}_{p}\left[Q_{i}\right]^{b}$ for suitable (non-negative) integers $a$ and $b$.

Now

$$
\operatorname{dim}_{\mathbb{Q}_{p}}\left(\mathbb{Q}_{p} \cdot D_{i+1}\right)=\operatorname{dim}_{\mathbb{Q}_{p}}\left(\mathbb{Q}_{p} \cdot A_{i+1}\right)-1=r_{L_{i+1}}+\delta_{L_{i+1}} \geq r_{L_{i+1}}=p \cdot r_{L_{i}}
$$

whilst, since (under hypothesis $\left.(*)_{i}\right)$ we are assuming that $L_{i}$ validates Leopoldt's Conjecture at $p$, one also has $\operatorname{dim}_{\mathbb{Q}_{p}}\left(\mathbb{Q}_{p} \cdot D_{i}\right)=r_{L_{i}}$. This gives an inequality

$$
\begin{aligned}
a+p \cdot b=\operatorname{dim}_{\mathbb{Q}_{p}}\left(\mathbb{Q}_{p} \cdot D_{i+1}\right) \geq p \cdot r_{L_{i}} \\
=p \cdot \operatorname{dim}_{\mathbb{Q}_{p}}\left(\mathbb{Q}_{p} \cdot D_{i}\right)=p \cdot \operatorname{dim}_{\mathbb{Q}_{p}}\left(\mathbb{Q}_{p} \cdot H_{0}\left(Q_{i}, D_{i+1}\right)\right)=p(a+b)
\end{aligned}
$$

and hence implies that $a=0$ and $b=r_{L_{i}}$. This shows both that $L_{i+1}$ validates Leopoldt's Conjecture at $p$ and also that $\overline{D_{i+1}}$ is a free $\mathbb{Z}_{p}\left[Q_{i}\right]$-module.

Then, since $\overline{D_{i+1}}$ is a free $\mathbb{Z}_{p}\left[Q_{i}\right]$-module, the tautological exact sequence

$$
0 \rightarrow\left(D_{i+1}\right)_{\text {tor }} \rightarrow D_{i+1} \rightarrow \overline{D_{i+1}} \rightarrow 0
$$

splits as a sequence of $\mathbb{Z}_{p}\left[Q_{i}\right]$-modules and so $H_{0}\left(Q_{i},\left(D_{i+1}\right)_{\text {tor }}\right)$ is isomorphic to a finite submodule of $H_{0}\left(Q_{i}, D_{i+1}\right)$. We have already shown the latter module to be torsion-free and so $H_{0}\left(Q_{i},\left(D_{i+1}\right)_{\text {tor }}\right)$ vanishes. By Nakayama's Lemma, this fact then implies that $\left(D_{i+1}\right)_{\text {tor }}$, and hence also $\left(A_{i+1}\right)_{\text {tor }}$, vanishes.

At this stage we have shown that the validity of hypothesis $(*)_{i}$ implies the validity of hypothesis $(*)_{i+1}$. 
Thus, since the stated assumptions on $L_{0}=K$ are equivalent to the validity of hypothesis $(*)_{0}$, an induction on $i$ shows that hypothesis $(*)_{i}$ is true for all integers $i$ with $0 \leq i \leq n$ and this fact is equivalent to the claimed result.

We can now prove Theorem 2.14. As a first step we choose a finite chain of subgroups $\{\mathrm{id}\}=J_{0} \unlhd J_{1} \unlhd \cdots \unlhd J_{n}=G$ in which $\left|J_{i+1} / J_{i}\right|=p$ for all $i$ with $0 \leq i<n$ (in doing so we can also ensure that, for any given intermediate field $E$ of $L / K$, one has $J_{i}=G_{L / E}$ for some $i$ ). For each such $i$ we set $L^{i}:=L^{J_{i}}$. Then by successively applying Proposition 5.1 to each of the extensions $L^{i} / L^{i+1}$ for $0 \leq i<n$ we deduce that $L$ validates Leopoldt's Conjecture at $p$ and that each group $A_{L^{i}}^{\Sigma}$ is torsion-free. In particular, this proves claim (i).

The first assertion of claim (ii) follows directly upon combining the fact that $L$ validates Leopoldt's Conjecture at $p$ with Corollary 3.2. Next we note that, since $B_{L}^{\Sigma}$ is torsion-free (as a subgroup of $A_{L}^{\Sigma}$ ), it is a free $\mathbb{Z}_{p}[G]$-module if and only if it is a cohomologically-trivial $G$-module (by [3, Chapter VI, Theorem (8.7)]). Thus, since the above argument shows that the group $G_{M_{E}^{\Sigma} / L^{\text {cyc }}}$ is torsion-free for every intermediate field $E$ of $L / K$, the second assertion of claim (ii) follows directly from Corollary 2.4.

Turning to claim (iii) (a) it is clear $\gamma^{|C|}$ belongs to $Z\left(G_{M_{L}^{\Sigma} / F}\right) \cap A_{L}^{\Sigma}$ and hence that the conjugation action of $C$ on $A_{L}^{\Sigma}$ induces an action on $D_{L}:=A_{L}^{\Sigma} /\left\langle\gamma^{|C|}\right\rangle$.

We show first that $D_{L}$ is a permutation module over $\mathbb{Z}_{p}[C]$. To do this we note that, as $D_{L}$ is torsion-free (by Proposition 5.1), the observation of Remark 4.2 shows it is enough to prove that for each subgroup $J$ of $C$ the group $\hat{H}^{-1}\left(J, D_{L}\right)$ vanishes and we recall that the latter groups were shown to vanish in the course of proving Proposition 5.1.

By a straightforward exercise one checks that a permutation module over $\mathbb{Z}_{p}[C]$ is free if and only if it spans a free $\mathbb{Q}_{p}[C]$-module and so claim (iii)(a) will follow if we can show that the $\mathbb{Q}_{p}[C]$-module $\mathbb{Q}_{p} \cdot D_{L}$ is free. To do this we note that the $\mathbb{Z}_{p}[C]$-module $\left\langle\gamma^{|C|}\right\rangle$ is isomorphic to $\mathbb{Z}_{p}$ (since $C$ acts trivially on $\gamma^{|C|}$ which has infinite order). Then, by comparing the tautological exact sequence

$$
0 \rightarrow\left\langle\gamma^{|C|}\right\rangle \rightarrow A_{L}^{\Sigma} \rightarrow D_{L} \rightarrow 0
$$

with the sequence (1), one deduces that the $\mathbb{Q}_{p}[C]$-module $\mathbb{Q}_{p} \cdot D_{L}$ is isomorphic to $\mathbb{Q}_{p} \cdot B_{L}^{\Sigma}$ and hence, by Corollary 3.2 , is free, as required.

The isomorphism in claim (iii)(b) is obtained by simply noting that $D_{L}$ is a free $\mathbb{Z}_{p}[C]$-module of rank $r_{F}=[G: C] \cdot r_{K}$ and hence that the exact sequence (11) splits.

Turning to claim (iii)(c) we note that the first assertion is an immediate consequence of the isomorphism in (iii)(b). We thus assume $r_{K}>0$. To derive the claimed exact sequence in this case we note first that $(1)$ induces a natural exact sequence of $\mathbb{Z}_{p}[C]$ modules

$$
0 \rightarrow\left\langle\gamma^{|C|}\right\rangle \cap B_{L}^{\Sigma} \rightarrow B_{L}^{\Sigma} \rightarrow D_{L} \rightarrow \Gamma_{L} /\langle\tilde{\gamma}\rangle \rightarrow 0
$$

where we write $\tilde{\gamma}$ for the image of $\gamma^{|C|}$ under the projection $A_{L}^{\Sigma} \rightarrow \Gamma_{L}$. Further, by claim (iii)(a) we know the $\mathbb{Z}_{p}[C]$-module $D_{L}$ is a free of $\operatorname{rank}[G: C] \cdot r_{K}$ and it is also clear that the $\mathbb{Z}_{p}[C]$-module $\left\langle\gamma^{|C|}\right\rangle \cap B_{L}^{\Sigma}$ is either isomorphic to $\mathbb{Z}_{p}$ or vanishes and that the 
element $\tilde{\gamma}$ is correspondingly either zero or non-zero. To discuss these possibilities we set $E:=L \cap F^{\text {cyc }}$.

We assume first that $E=F$, and hence that $L$ and $F^{\text {cyc }}$ are disjoint over $F$. Since the $\mathbb{Z}_{p}$-rank of $A_{L}^{\Sigma}$ is $|G| \cdot r_{K}+1>1$, we may therefore in this case choose the element $\gamma$ so that $\tilde{\gamma}$ vanishes. After choosing $\gamma$ in this way, the sequence (12) directly gives the exact sequence in claim (iii)(c) with $n_{C}=0$.

We assume next that $E \neq F$. In this case the restriction of $\gamma$ to $E$ generates the non-trivial quotient $G_{E / F}$ of $\Gamma_{F}$ and so the restriction of $\gamma$ to $F^{\text {cyc }}$ must generate $\Gamma_{F}$. The image of $\tilde{\gamma}$ in $\Gamma_{F}$ therefore generates $\left(\Gamma_{F}\right)^{|C|}$ and hence, since $\Gamma_{L}$ identifies with $\left(\Gamma_{F}\right)^{[E: F]}$, one has $\langle\tilde{\gamma}\rangle=\left(\Gamma_{L}\right)^{[L: E]}$ and so the $\mathbb{Z}_{p}[C]$-module $\Gamma_{L} /\langle\tilde{\gamma}\rangle$ is isomorphic to $\mathbb{Z}_{p} /\left(\mathbb{Z}_{p} \cdot[L: E]\right)$. Given these observations, the exact sequence in claim (iii)(c) (with $\left.n_{C}=1\right)$ again follows directly from (12).

This completes the proof of Theorem 2.14.

\section{The proof of Theorem 2.17.}

In this section we fix an odd prime $p$ that does not divide $h_{\mathbb{Q}\left(\zeta_{p}\right)^{+}}$.

We adopt the general notation of Section 2.5. In addition, for each natural number $n$ we abbreviate the groups $A_{L_{n}}^{p}$ and $B_{L_{n}}^{p}$ to $A_{n}$ and $B_{n}$ respectively, we set $R_{n}:=\mathbb{Z}_{p}\left[G_{n}\right]$ and for any homomorphism $\phi$ in $H_{n}^{*}$ and any $R_{n}$-module $M$ we set $M^{\phi}:=e_{\phi}(M)$ and regard this as a module over the ring $R_{n}^{\phi}$ in the natural way.

\subsection{The proof of Theorem 2.17(i).}

At the outset we recall that $L_{n}$, and hence also $L_{n}^{+}$, validates Leopoldt's Conjecture at $p$.

The first claimed isomorphism of $R_{n}$-modules $A_{n} \cong B_{n} \oplus \mathbb{Z}_{p}$ is thus a consequence of Corollary 2.4 with $L=L_{n}, K=\mathbb{Q}$ and $\Sigma=\{\infty, p\}$.

To prove $\overline{B_{n}}$ is a free $R_{n}^{-}$-module we show first that $\left(\overline{B_{n}}\right)^{+}$vanishes. To do this we note the maximal abelian extension of $L_{n}^{+}$in $M_{L_{n}}^{p}$ is $E:=L_{n} \cdot M_{L_{n}^{+}}^{p}$. This implies $A_{n}^{+}=G_{E / L_{n}}$ and hence that $B_{n}^{+}$is equal to $G_{E / L_{n}^{\text {cyc }}}$ which is naturally isomorphic to $G_{M_{L_{n}^{+}}^{p} /\left(L_{n}^{+}\right)^{\text {cyc }}}=B_{L_{n}^{+}}^{p}$. Thus, since $L_{n}^{+}$is totally real and validates Leopoldt's Conjecture, the group $\left(\overline{B_{n}}\right)^{+}=\overline{B_{n}^{+}} \cong \overline{B_{L_{n}^{+}}^{p}}$ vanishes, as required.

We now need to prove that $\overline{B_{n}}=\left(\overline{B_{n}}\right)^{-}$is a free $R_{n}^{-}$-module of rank one. To do this we note $\mathbb{Z}_{p} \otimes_{\mathbb{Z}} W_{L_{n}}$ is a free rank one $R_{n}^{-}$-module, where $W_{L_{n}}$ is as defined at the beginning of Section 3.1, and hence that for each $\phi$ in $H_{n}^{*,-}$ Proposition 3.1(iv) implies the $\mathbb{Z}_{p}$-ranks of $R_{n}^{\phi}$ and $\left(\overline{B_{n}}\right)^{\phi}$ are equal. Since each ring $R_{n}^{\phi}$ is local it is thus enough to show that $\overline{B_{n}}$ is a free $\mathbb{Z}_{p}\left[P_{n}\right]$-module, or equivalently (by $[\mathbf{3}$, Chapter VI, Theorem (8.7)]) that $\overline{B_{n}}$ is a cohomologically-trivial $P_{n}$-module.

By the same reduction argument used in the proof of Corollary 2.4, it is thus enough to prove that the group $\hat{H}^{-1}\left(J, \overline{B_{n}}\right)$ vanishes for each subgroup $J$ of $P_{n}$ and this follows directly from Lemma 6.1 below. This completes the proof of Theorem 2.17(i).

In the sequel, for a number field $F$ we write $P_{F}$ and $H_{F}$ for the subgroups of $F^{\times}$ comprising $p$-units and those elements $x$ for which the extension $F(\sqrt[p]{x})$ is unramified outside $p$ respectively, and $\mathrm{Cl}_{F}^{p}$ for the quotient of $\mathrm{Cl}_{F}$ by the subgroup generated by 
the classes of ideals above $p$. We use the fact that these groups are related by a natural exact sequence

$$
0 \rightarrow P_{F} / P_{F}^{p} \rightarrow H_{F}\left(F^{\times}\right)^{p} /\left(F^{\times}\right)^{p} \stackrel{\Delta_{F}}{\longrightarrow} \mathrm{Cl}_{F,[p]}^{p} \rightarrow 0
$$

(see, for example, the proof of [1, Proposition 2.4]).

LEMMA 6.1. If $p$ does not divide $h_{\mathbb{Q}\left(\zeta_{p}\right)^{+}}$, then for each integer a with $1 \leq a \leq n$ the group $\hat{H}^{-1}\left(G_{L_{n} / L_{a}}, \overline{B_{n}}\right)$ vanishes.

Proof. If $n=1$, this result is obvious because $G_{L_{n} / L_{a}}$ is the trivial group.

If $n>1$, then, as already observed above, the exact sequence (1) splits and so the description of Lemma 4.1 shows it is enough to prove $T_{L_{n} / L_{a}}^{p}$ vanishes. Recalling Lemma 4.3(iii), it therefore suffices to show that for any degree $p$ Galois extension $E$ of $L_{a}$ that is not $\mathbb{Z}_{p}$-extendable the extension $L_{n} E / L_{n}$ is not $\mathbb{Z}_{p}$-extendable.

To check this condition we use the fact that if $F$ is either $L_{n}$ or $L_{a}$ and $x$ is any element of $F^{\times}$, then Bertrandias and Payan [1, Proposition 2.7] have shown that $F(\sqrt[p]{x}) / F$ is $\mathbb{Z}_{p}$-extendable if and only if $x$ belongs to $P_{F}\left(F^{\times}\right)^{p}$. From the sequence (13) it is thus enough to show that if $x$ is any element of $L_{a}^{\times}$with $\Delta_{L_{a}}(x) \neq 0$, then also $\Delta_{L_{n}}(x) \neq 0$.

Since there is a commutative diagram

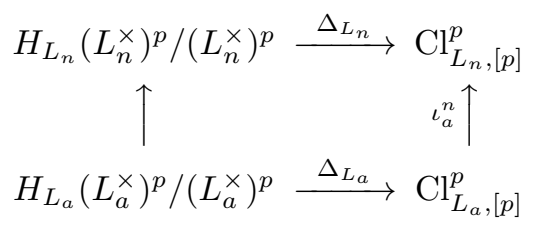

where the left hand vertical map is induced by the inclusion $L_{a} \subseteq L_{n}$ and $\iota_{a}^{n}$ is the natural inflation, it thus suffices to show $\iota_{a}^{n}$ is injective.

This is true because for $b \in\{n, a\}$ one has $\mathrm{Cl}_{L_{b},[p]}^{p}=\mathrm{Cl}_{L_{b},[p]}$ (as all prime ideals of $L_{b}$ above $p$ are principal), because $\mathrm{Cl}_{L_{b},[p]}^{+}$vanishes (by the stated assumption on $p$ ) and because the inflation map $\mathrm{Cl}_{L_{a},[p]}^{-} \rightarrow \mathrm{Cl}_{L_{n},[p]}^{-}$is injective (as is shown, for example, by Kida in $[\mathbf{1 9}$, Proposition 1, (1.2)]).

\subsection{The proof of Theorem 2.17 (ii).}

The key fact that we use in the proof of Theorem 2.17(ii) is the known validity (due to Flach and the first author) of the appropriate case of the equivariant Tamagawa number conjecture. However, we must also first prove several auxiliary results.

We recall that for each integer $i$ the ring $e_{\omega^{i}} R_{n}$ is abbreviated to $R_{n}^{(i)}$. For convenience we also write $e_{n}$ for the idempotent $e_{\omega^{0}}=e_{H_{n}}$ of $\mathbb{Z}_{p}\left[H_{n}\right]$.

\subsubsection{A useful exact triangle.}

At the outset we fix, as we may, an element $\gamma$ of $G_{M_{L_{n}}^{p} / \mathbb{Q}}$ which projects to give both the element $\gamma_{n}$ of $G_{n}$ and the element $\gamma_{\mathbb{Q}}$ of $\Gamma_{\mathbb{Q}}$ that are fixed at the beginning of Section 2.5.3.

We then write $C_{n, \gamma}^{\bullet}$ for the complex of projective $R_{n}$-modules 


$$
R_{n}^{(0)} \stackrel{d_{\gamma}}{\longrightarrow} R_{n}^{(0)}
$$

where the first term is placed in degree two and $d_{\gamma}$ satisfies $d_{\gamma}\left(e_{n}\right)=\left(1-\gamma_{n}\right) e_{n}$. We identify the groups $H^{2}\left(C_{n, \gamma}^{\bullet}\right)=\operatorname{ker}\left(d_{\gamma}\right)$ and $H^{3}\left(C_{n, \gamma}^{\bullet}\right)=\operatorname{cok}\left(d_{\gamma}\right)$ with $\mathbb{Z}_{p}$ via the maps of $R_{n}$-modules $\iota: \mathbb{Z}_{p} \rightarrow R_{n}^{(0)}$ and $\epsilon: R_{n}^{(0)} \rightarrow \mathbb{Z}_{p}$ with $\iota(1)=\sum_{g \in P_{n}} g e_{n}$ and $\epsilon\left(g e_{n}\right)=1$ for all $g \in G_{n}$.

Finally we set $W_{n, p}:=\mathbb{Z}_{p} \otimes_{\mathbb{Z}} W_{L_{n}}$ and write $C_{n}^{\bullet}$ for the complex

$$
W_{n, p}[-1] \oplus R_{n}^{-}[-2] .
$$

The following result refines the description of $R \Gamma_{c, \text { ét }}\left(\mathcal{O}_{L_{n}, \Sigma_{p}}, \mathbb{Z}_{p}(1)\right)$ given in Proposition 3.1.

Proposition 6.2. Assume $p$ does not divide $h_{\mathbb{Q}\left(\zeta_{p}\right)^{+}}$and fix an element $b$ of $B_{n}^{-}=$ $A_{n}^{-}$that projects to give a basis of the (free, rank one) $R_{n}^{-}$-module $\overline{B_{n}}$. Then there exists a canonical exact triangle in $D^{\text {perf }}\left(R_{n}\right)$ of the form

$$
C_{n, \gamma}^{\bullet} \oplus C_{n}^{\bullet} \stackrel{\theta_{\gamma} \oplus \theta_{b}}{\longrightarrow} R \Gamma_{c, \text { ét }}\left(\mathcal{O}_{L_{n}, \Sigma_{p}}, \mathbb{Z}_{p}(1)\right) \rightarrow A_{n, \text { tor }}[-2] \rightarrow\left(C_{n, \gamma}^{\bullet} \oplus C_{b}^{\bullet}\right)[1]
$$

in which $H^{2}\left(\theta_{\gamma}\right)(1)=\gamma^{\left|G_{n}\right|} \in A_{n}, H^{3}\left(\theta_{\gamma}\right)$ is the identity on $\mathbb{Z}_{p}, H^{1}\left(\theta_{b}\right)$ is the canonical identification $W_{n, p}=H_{c, \text { ét }}^{1}\left(\mathcal{O}_{L_{n}, \Sigma_{p}}, \mathbb{Z}_{p}(1)\right.$ ) (see Proposition $3.1(\mathrm{iv})$ ) and $H^{2}\left(\theta_{b}\right)$ sends each element $x$ of $\mathbb{Z}_{p}\left[G_{n}\right]^{-}$to $x(b)$.

In particular, the $G_{n}$-module $A_{n, \text { tor }}$ is cohomologically-trivial.

Proof. Set $E_{n}^{\bullet}:=R \Gamma_{c, \text { ét }}\left(\mathcal{O}_{L_{n}, \Sigma_{p}}, \mathbb{Z}_{p}(1)\right)$.

Then all of the complexes $C_{n, \gamma}^{\bullet}, C_{n}^{\bullet}$ and $E_{n}^{\bullet}$ belong to $D^{\text {perf }}\left(R_{n}\right)$. In the first case this is clear, in the second it is true because $W_{n, p}$ is a free $R_{n}^{-}$-module of rank one and in the third case it follows from Proposition 3.1(i).

The descriptions of the cohomology of $E_{n}^{\bullet}$ given in Proposition 3.1(iii) then make it clear that there exists a unique morphism $\theta_{b}: C_{n}^{\bullet} \rightarrow E_{n}^{\bullet}$ in $D\left(R_{n}\right)$ with the given descriptions of $H^{1}\left(\theta_{b}\right)$ and $H^{2}\left(\theta_{b}\right)$. In addition, the construction of a morphism $\theta_{\gamma}$ : $C_{n, \gamma}^{\bullet} \rightarrow E_{n}^{\bullet}$ in $D^{\text {perf }}\left(R_{n}\right)$ with the given descriptions of $H^{2}\left(\theta_{\gamma}\right)$ and $H^{3}\left(\theta_{\gamma}\right)$ is described by Macias Castillo and the first author in [8, Proposition 4.3].

Writing $D_{n}^{\bullet}$ for the mapping cone of $\theta_{\gamma} \oplus \theta_{b}$, it therefore suffices to show that the long exact cohomology sequence of this cone implies it is acyclic outside degree two and such that the $R_{n}$-module $H^{2}\left(D_{n}^{\bullet}\right)$ is naturally isomorphic to $A_{n, \text { tor }}$.

This long exact sequence makes the vanishing of $H^{i}\left(D_{n}^{\bullet}\right)$ for $i \notin\{0,1,2,3\}$ immediately clear and shows that the vanishing of $H^{0}\left(D_{n}^{\bullet}\right)$ and $H^{3}\left(D_{n}^{\bullet}\right)$ follows directly from the (obvious) injectivity of $H^{1}\left(\theta_{b}\right)$ and surjectivity of $H^{3}\left(\theta_{\gamma}\right)$ respectively, that the vanishing of $H^{1}\left(D_{n}^{\bullet}\right)$ follows from the (obvious) surjectivity of $H^{1}\left(\theta_{b}\right)$ and injectivity of both $H^{2}\left(\theta_{\gamma}\right)$ and $H^{2}\left(\theta_{b}\right)$ and that the (obvious) injectivity of $H^{3}\left(\theta_{\gamma}\right)$ gives rise to a short exact sequence

$$
0 \rightarrow \operatorname{im}\left(H^{2}\left(\theta_{\gamma} \oplus \theta_{b}\right)\right) \rightarrow A_{n} \rightarrow H^{2}\left(D_{n}^{\bullet}\right) \rightarrow 0 .
$$

Now the group $\operatorname{im}\left(H^{2}\left(\theta_{\gamma} \oplus \theta_{b}\right)\right)=\operatorname{im}\left(H^{2}\left(\theta_{\gamma}\right)\right)+\operatorname{im}\left(H^{2}\left(\theta_{b}\right)\right)$ is $\mathbb{Z}_{p}$-free and so disjoint 
from $A_{n \text {,tor }}=B_{n \text {,tor }}$. Since the element $H^{2}\left(\theta_{\gamma}\right)(1)=\gamma^{\left|G_{n}\right|}$ is a topological generator of $\Gamma_{L_{n}}$ and our choice of element $b$ implies $B_{n \text {,tor }}+\operatorname{im}\left(H^{2}\left(\theta_{b}\right)\right)=B_{n}$, we therefore obtain a direct sum decomposition $A_{n}=A_{n \text {, tor }} \oplus \operatorname{im}\left(H^{2}\left(\theta_{\gamma} \oplus \theta_{b}\right)\right)$. Given this, the exact sequence (14) induces a natural isomorphism of $R_{n}$-modules $H^{2}\left(D_{n}^{\bullet} \cong A_{n \text {,tor }}\right.$, as required.

Finally we note that the given exact triangle implies $A_{n, \text { tor }}[-2]$ belongs to $D^{\text {perf }}\left(R_{n}\right)$. This in turn implies that the $R_{n}$-module $A_{n \text {,tor }}$ has finite projective dimension and hence that it is cohomologically-trivial over $G_{n}$, as claimed.

REMARK 6.3. It will be seen later (in Lemma 6.5) that if $p$ does not divide $h_{\mathbb{Q}\left(\zeta_{p}\right)^{+}}$, then $A_{n, \text { tor }}^{-}$vanishes. Thus, since $C_{n, \gamma}^{\bullet,--}$ is clearly the zero complex, in this case the exact triangle in Proposition 6.2 induces an isomorphism in $D^{\text {perf }}\left(\mathbb{Z}_{p}\left[G_{n}\right]\right)$ between $R \Gamma_{c \text {,ét }}\left(\mathcal{O}_{L_{n}, \Sigma_{p}}, \mathbb{Z}_{p}(1)\right)^{-}$and the explicit complex $C_{n}^{\bullet}=\left(C_{n}^{\bullet}\right)^{-}$.

\subsubsection{The Fitting ideal.}

In this section we combine the exact triangle constructed in Proposition 6.2 with the known validity, due to Flach and the first author, of the equivariant Tamagawa number conjecture for the pair $\left(h^{0}\left(\operatorname{Spec}\left(L_{n}\right)\right)(1), \mathbb{Z}\left[G_{n}\right]\right)$ to prove the following result.

TheOREM 6.4. Fix an element $u$ of $\left(\mathbb{Z}_{p} \hat{\otimes} L_{n, p}^{\times}\right)^{-}$as in the statement of Theorem 2.17(iv) and set

$$
\mathcal{L} \mathcal{R}_{u}:=\sum_{\chi \in G_{n}^{*,-}} \mathcal{L} \mathcal{R}_{u}^{\chi} \cdot e_{\chi} \in \mathbb{C}_{p}\left[G_{n}\right]
$$

Then $\theta_{n}^{*}(1)\left(\mathcal{L} \mathcal{R}_{u}+\epsilon_{\gamma_{\mathbb{Q}}}^{n} \mathcal{L}_{\infty, p}^{n}\right)$ belongs to $R_{n}$ and generates the ideal $\operatorname{Fit}_{R_{n}}\left(A_{n, \text { tor }}\right)$.

Proof. For any commutative noetherian ring $R$ we write $\operatorname{Det}_{R}(-)$ for the determinant functor defined by Knudsen and Mumford in [20]. We recall that this functor is well-defined on the category $D^{\text {perf }}(R)$ and takes values in the category of graded invertible $R$-modules.

If $R$ is semisimple, $C$ is an object of $D^{\text {perf }}(R)$ that is acyclic outside degrees $i$ and $i+1$ for some integer $i$ and $\mu$ is an isomorphism of $R$-modules $H^{i}(C) \cong H^{i+1}(C)$, then we write $\vartheta_{\mu}$ for the composite isomorphism

$$
\begin{aligned}
\operatorname{Det}_{R}(C) & \cong \operatorname{Det}_{R}\left(H^{i}(C)\right)^{(-1)^{i}} \otimes \operatorname{Det}_{R}\left(H^{i+1}(C)\right)^{(-1)^{i+1}} \\
& \cong \operatorname{Det}_{R}\left(H^{i+1}(C)\right)^{(-1)^{i}} \otimes \operatorname{Det}_{R}\left(H^{i+1}(C)\right)^{(-1)^{i+1}} \\
& \cong(R, 0)
\end{aligned}
$$

where the first map is the canonical 'passage to cohomology' isomorphism (which exists since $R$ is semisimple), the second is $\operatorname{Det}_{R}(\mu)^{(-1)^{i}} \otimes$ id and the third is induced by the evaluation pairing on $H^{i+1}(C)$.

To prove the stated result we set $E_{n}^{\bullet}:=R \Gamma_{c \text {,ét }}\left(\mathcal{O}_{L_{n}, \Sigma_{p}}, \mathbb{Z}_{p}(1)\right)$ and use, without explicit comment, the descriptions of the cohomology groups $H^{i}\left(E_{n}^{\bullet}\right)$ that are given in Proposition 3.1. We also write $\Lambda_{n}$ for the semisimple algebra $\mathbb{C}_{p} \cdot R_{n}$.

It clearly suffices to show both that $\left(\theta_{n}^{*}(1) \mathcal{L} \mathcal{R}_{u}\right) R_{n}^{-}=\operatorname{Fit}_{R_{n}^{-}}\left(A_{n, \text { tor }}^{-}\right)$and that $\left(\theta_{n}^{*}(1) \epsilon_{\gamma_{\mathbb{Q}}}^{n} \mathcal{L}_{\infty, p}^{n}\right) R_{n}^{+}=\operatorname{Fit}_{R_{n}^{+}}\left(A_{n, \text { tor }}^{+}\right)$. 
To prove the first equality we note that the proof of Castillo and Jones [9, Proposition 2.2] (which depends on the result of Flach and the first author) describes an explicit isomorphism of $\Lambda_{n}$-modules $\Phi_{n}: \mathbb{C}_{p} \cdot H^{2}\left(E_{n}^{\bullet}\right)^{-} \cong \mathbb{C}_{p} \cdot H^{1}\left(E_{n}^{\bullet}\right)^{-}$for which there is an equality of graded invertible $R_{n}^{-}$-modules

$$
\left(\theta_{n}^{*}(1) \cdot R_{n}^{-}, 0\right)=\vartheta_{\Phi_{n}^{-1}}\left(\operatorname{Det}_{R_{n}^{-}}\left(E_{n}^{\bullet,-}\right)\right)^{-1}
$$

To explicate this equality we write $\epsilon_{u}$ for the homomorphism of $R_{n}$-modules $H^{1}\left(E_{n}^{\bullet}\right)=W_{n, p} \rightarrow H^{2}\left(E_{n}^{\bullet}\right)$ which sends the $R_{n}$-generator

$$
w_{n}:=2 \pi i \cdot\left(\sigma_{n}-\sigma_{n} \circ \tau_{n}\right)
$$

of $W_{n, p}$ to the image of the chosen element $u$ under the global reciprocity map $\left(\mathbb{Z}_{p} \hat{\otimes} L_{n, p}^{\times}\right)^{-} \rightarrow B_{n}^{-}=A_{n}^{-}=H^{2}\left(E_{n}^{\bullet}\right)^{-}$. Then an explicit comparison of the result of $\left[\mathbf{9}\right.$, Proposition 2.3] with our definition of the equivariant resolvent $\mathcal{L} \mathcal{R}_{u}$ implies that

$$
\operatorname{det}_{\Lambda_{n}}\left(\Phi_{n} \circ\left(\mathbb{C}_{p} \otimes_{\mathbb{Z}_{p}} \epsilon_{u}\right)\right)=\mathcal{L} \mathcal{R}_{u}
$$

Since our choice of $u$ implies that the map $\mathbb{C}_{p} \otimes \epsilon_{u}$ is invertible, this equality combines with (15) to give an equality of graded invertible $R_{n}^{-}$-modules

$$
\left(\left(\theta_{n}^{*}(1) \cdot \mathcal{L} \mathcal{R}_{u}\right) R_{n}^{-}, 0\right)=\vartheta_{\mathbb{C}_{p} \otimes \epsilon_{u}}\left(\operatorname{Det}_{R_{n}^{-}}\left(E_{n}^{\bullet,-}\right)\right)^{-1} .
$$

To compute the right hand side of this equality we use the exact triangle constructed in Proposition 6.2 in the case that the element $b$ is chosen to have the same image in $\overline{B_{n}}$ as does the image of $u$ under the reciprocity map. In this case the image of $H^{2}\left(\theta_{b}\right)\left(\left(1-\tau_{n}\right) / 2\right)$ in $\overline{B_{n}}$ is equal to the image of $H^{1}\left(\theta_{b}\right)\left(w_{n}\right)$ under $\mathbb{C}_{p} \otimes \epsilon_{u}$. Thus, if we write $\mu_{b}$ for the isomorphism of (free rank one) $R_{n}^{-}$-modules $W_{n, p} \rightarrow R_{n}^{-}$which sends $w_{n}$ to $\left(1-\tau_{n}\right) / 2$, then one has

$$
\begin{aligned}
\vartheta_{\mathbb{C}_{p} \otimes \epsilon_{u}}\left(\operatorname{Det}_{R_{n}^{-}}\left(E_{n}^{\bullet,-}\right)\right) & =\vartheta_{\mathbb{C}_{p} \otimes \mu_{b}}\left(\operatorname{Det}_{R_{n}^{-}}\left(C_{n}^{\bullet}\right)\right) \otimes \operatorname{Det}_{R_{n}^{-}}\left(A_{n, \text { tor }}^{-}[-2]\right) \\
& =\operatorname{Det}_{R_{n}^{-}}\left(A_{n, \text { tor }}^{-}[-2]\right) \\
& =\left(\operatorname{Fit}_{R_{n}^{-}}\left(A_{n, \text { tor }}^{-}\right)^{-1}, 0\right) .
\end{aligned}
$$

Here the first equality follows from the exact triangle in Proposition 6.2 and the fact that $C_{n, \gamma}^{\bullet,-}$ is the zero complex; the second equality in (17) is true because

$$
\begin{aligned}
\vartheta_{\mathbb{C}_{p} \otimes \mu_{b}}\left(\operatorname{Det}_{R_{n}^{-}}\left(C_{n}^{\bullet}\right)\right) & =\left(\vartheta_{\mathrm{ev}} \circ\left(\operatorname{Det}_{R}\left(\mu_{b}\right)^{-1} \otimes \mathrm{id}\right)\right)\left(\left(\operatorname{Hom}_{R_{n}^{-}}\left(W_{n, p}, R_{n}^{-}\right),-1\right) \otimes\left(R_{n}^{-}, 1\right)\right) \\
& =\vartheta_{\mathrm{ev}}\left(\left(\operatorname{Hom}_{R_{n}^{-}}\left(R_{n}^{-}, R_{n}^{-}\right),-1\right) \otimes\left(R_{n}^{-}, 1\right)\right) \\
& =\left(R_{n}^{-}, 0\right)
\end{aligned}
$$

where $\vartheta_{\text {ev }}$ is induced by the evaluation pairing on $R_{n}^{-}$and the second equality follows from the fact that $\mu_{b}\left(W_{n, p}\right)=R_{n}^{-}$; the final equality in (17) follows from a general property of Fitting ideals since Proposition 6.2 implies that the finite $R_{n}^{-}$-module $A_{n \text {,tor }}^{-}$ is of projective dimension at most one.

The equalities (16) and (17) then combine to imply that $\left(\theta_{n}^{*}(1) \cdot \mathcal{L} \mathcal{R}_{u}\right) R_{n}^{-}$is equal 
to Fit $_{R_{n}^{-}}\left(A_{n, \text { tor }}^{-}\right)$, as required.

To prove $\left(\theta_{n}^{*}(1) \epsilon_{\gamma_{Q}}^{n} \mathcal{L}_{\infty, p}^{n}\right) R_{n}^{+}=\operatorname{Fit}_{R_{n}^{+}}\left(A_{n, \text { tor }}^{+}\right)$we note first that the homomorphisms $\log _{\infty}^{n}$ and $\log _{p}^{n}$ in Section 2.5.2 combine to give an isomorphism of $\mathbb{C}_{p}\left[G_{n}\right]$-modules

$$
\log _{\infty, p}^{n}: \mathbb{C}_{p} \otimes_{\mathbb{Q}} L_{n, 0}^{+} \stackrel{\left(\mathbb{C}_{p} \cdot \log _{\infty}^{n}\right)^{-1}}{\longrightarrow} \mathbb{C}_{p} \otimes_{\mathbb{Z}} \mathcal{O}_{L_{n}^{+}}^{\times} \stackrel{\mathbb{C}_{p} \cdot \log _{p}^{n}}{\longrightarrow} \mathbb{C}_{p} \otimes_{\mathbb{Q}} L_{n, 0}^{+},
$$

and that, by the very definition of the $\mathcal{L}$-invariant $\mathcal{L}_{\infty, p}^{n}$, one has

$$
\mathcal{L}_{\infty, p}^{n}=\operatorname{det}_{\mathbb{C}_{p}\left[G_{n}\right]}\left(\log _{\infty, p}^{n}\right) .
$$

We also note that $E_{n}^{\bullet,+}$ is acyclic outside degrees two and three and that there is a canonical composite isomorphism of $\mathbb{Q}_{p}\left[G_{n}\right]$-modules

$$
\Xi_{n}: \mathbb{Q}_{p} \cdot H^{2}\left(E_{n}^{\bullet,+}\right)=\mathbb{Q}_{p} \cdot \Gamma_{L_{n}^{+}} \stackrel{\log _{p} \circ \chi_{\mathbb{Q}}}{\longrightarrow} \mathbb{Q}_{p}=\mathbb{Q}_{p} \cdot H^{3}\left(E_{n}^{\bullet,+}\right) .
$$

Then, in terms of this notation, the validity of the equivariant Tamagawa number conjecture for $\left(h^{0}\left(\operatorname{Spec}\left(L_{n}^{+}\right)\right)(1), \mathbb{Z}\left[G_{n}\right]^{+}\right)$implies that

$$
\left(\theta_{n}^{*}(1) \mathcal{L}_{\infty, p}^{n} \cdot R_{n}^{+}, 0\right)=\vartheta_{\Xi_{n}}\left(\operatorname{Det}_{R_{n}^{+}}\left(E_{n}^{\bullet,+}\right)\right)^{-1} .
$$

(This equality follows directly from the explicit computation of Breuning and the first author in [2, Section 5.3] after taking into account (18) and the fact that the isomorphism $\mathbb{Q}_{p} \cdot \mathbb{Z}_{p}^{\times} \cong \mathbb{Q}_{p} \cdot \Gamma_{\mathbb{Q}}$ induced by the reciprocity map is the inverse of the isomorphism $\mathbb{Q}_{p} \cdot \Gamma_{\mathbb{Q}} \cong \mathbb{Q}_{p} \cdot \mathbb{Z}_{p}^{\times}$induced by $\left.\chi_{\mathbb{Q}} \cdot\right)$

In addition the exact triangle in Proposition 6.2 implies that

$$
\vartheta_{\Xi_{n}}\left(\operatorname{Det}_{R_{n}^{+}}\left(E_{n}^{\bullet,+}\right)\right)=\vartheta_{\Xi_{n}^{\gamma}}\left(\operatorname{Det}_{R_{n}^{+}}\left(C_{n, \gamma}^{\bullet}\right)\right) \otimes \operatorname{Det}_{R_{n}^{+}}\left(A_{n, \text { tor }}^{+}[-2]\right)
$$

where we write $\Xi_{n}^{\gamma}$ for the composite isomorphism

$$
\mathbb{Q}_{p} \cdot H^{2}\left(C_{n, \gamma}^{\bullet}\right) \stackrel{\mathbb{Q}_{p} \otimes_{\mathbb{Z}_{p}} H^{2}\left(\theta_{\gamma}\right)}{\longrightarrow} \mathbb{Q}_{p} \cdot H^{2}\left(E_{n}^{\bullet,+}\right) \stackrel{\Xi_{n}}{\longrightarrow} \mathbb{Q}_{p} \cdot H^{3}\left(E_{n}^{\bullet,+}\right)=\mathbb{Q}_{p} \cdot H^{3}\left(C_{n, \gamma}^{\bullet}\right) .
$$

It now suffices to prove that

$$
\vartheta_{\Xi_{n}^{\gamma}}\left(\operatorname{Det}_{R_{n}}\left(C_{n, \gamma}^{\bullet}\right)\right)=\left(\left(\epsilon_{\gamma_{\mathbb{Q}}}^{n}\right)^{-1} R_{n}^{+}, 0\right),
$$

since, if this is true, then it combines with (19) and (20) to directly imply the required equality

$$
\left(\theta_{n}^{*}(1) \epsilon_{\gamma_{\mathbb{Q}}}^{n} \mathcal{L}_{\infty, p}^{n} \cdot R_{n}^{+}, 0\right)=\operatorname{Det}_{R_{n}^{+}}\left(A_{n, \text { tor }}^{+}[-2]\right)^{-1}=\left(\operatorname{Fit}_{R_{n}^{+}}\left(A_{n, \text { tor }}^{+}\right), 0\right)
$$

(where the last equality follows in just the same way as the last equality of (17)).

It is enough to prove the $\omega^{a}$-component of (21) for every even integer $a$ with $0 \leq$ $a \leq p-2$. Further, if $a \neq 0$, then $C_{n, \gamma}^{\bullet,(a)}$ is the zero complex and $e_{\omega^{a}} \epsilon_{\gamma}^{n}=\epsilon_{\gamma}^{\omega^{a}} e_{\omega^{a}}=e_{\omega^{a}}$ and so the $\omega^{a}$-component of the equality is clear.

To compute $\vartheta_{\Xi_{n}^{\gamma}}\left(\operatorname{Det}_{R_{n}}\left(C_{n, \gamma}^{\bullet}\right)\right)^{(0)}$ we note that $\operatorname{Det}_{R_{n}^{(0)}}\left(C_{n, \gamma}^{\bullet,(0)}\right)$ is generated over $R_{n}^{(0)}$ 
by $x:=\left(e_{n}, 1\right) \otimes\left(e_{n}^{*},-1\right)$ where $e_{n}^{*}$ is the dual of $e_{n}$ in $\operatorname{Hom}_{R_{n}^{(0)}}\left(R_{n}^{(0)}, R_{n}^{(0)}\right)$.

Now, since $e_{n}=e_{G_{n}}+\left(e_{n}-e_{G_{n}}\right), \mathbb{Q}_{p} \cdot H^{2}\left(C_{n, \gamma}^{\bullet}\right)=\mathbb{Q}_{p} \cdot e_{G_{n}}, d_{\gamma}\left(e_{n}-e_{G_{n}}\right)=\left(1-\gamma_{n}\right) e_{n}$ and $\Xi_{n}^{\gamma}\left(e_{G_{n}}\right)=\log _{p}\left(\chi_{\mathbb{Q}}(\gamma)\right)=\log _{p}\left(\chi_{\mathbb{Q}}\left(\gamma_{\mathbb{Q}}\right)\right)$ one has

$$
\begin{aligned}
\vartheta_{\Xi_{n}^{\gamma}}(x) & =\left(\left(1-\gamma_{n}\right) e_{n}, 0\right)+\left(\vartheta_{\mathrm{ev}}^{\prime} \circ\left(\operatorname{Det}_{\mathbb{Q}_{p} \cdot R_{n}^{+}}\left(\Xi_{n}^{\gamma}\right) \otimes \mathrm{id}\right)\right)\left(\left(e_{G_{n}}, 1\right) \otimes\left(e_{G_{n}}^{*},-1\right)\right) \\
& =\left(\left(1-\gamma_{n}\right) e_{n}, 0\right)+\vartheta_{\mathrm{ev}}^{\prime}\left(\left(\log _{p}\left(\chi_{\mathbb{Q}}\left(\gamma_{\mathbb{Q}}\right)\right) e_{G_{n}}, 1\right) \otimes\left(e_{G_{n}}^{*},-1\right)\right) \\
& =\left(\left(1-\gamma_{n}\right) e_{n}+\log _{p}\left(\chi_{\mathbb{Q}}\left(\gamma_{\mathbb{Q}}\right)\right) e_{G_{n}}, 0\right) \\
& =\left(e_{n}\left(\epsilon_{\gamma_{\mathbb{Q}}}^{n}\right)^{-1}, 0\right)
\end{aligned}
$$

where $\vartheta_{\mathrm{ev}}^{\prime}$ is induced by the evaluation pairing on $\mathbb{Q}_{p} \cdot e_{G_{n}}, e_{G_{n}}^{*}$ denotes the dual of $e_{G_{n}}$ in $\operatorname{Hom}_{\mathbb{Q}_{p}}\left(\mathbb{Q}_{p} \cdot e_{G_{n}}, \mathbb{Q}_{p}\right)$ and the last equality follows by explicit comparison with the definition of $\epsilon_{\gamma_{\mathbb{Q}}}^{n}$. This completes the proof of Theorem 6.4.

\subsubsection{Completion of the proof.}

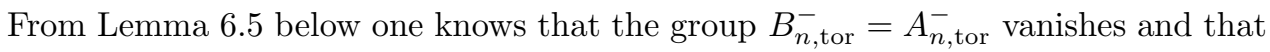
the $R_{n}$-module $A_{n, \text { tor }}=B_{n \text {,tor }}^{+}=B_{n}^{+}$is cyclic.

By a general property of Fitting ideals the latter fact implies $\operatorname{Fit}_{R_{n}}\left(A_{n, \text { tor }}\right)$ is equal to $\operatorname{Ann}_{R_{n}}\left(A_{n, \text { tor }}\right)$ and hence also then combines with the formula of Theorem 6.4 to imply that the $R_{n}$-module $A_{n \text {,tor }}=A_{n \text {,tor }}^{+}$is isomorphic to

$$
\left(R_{n} /\left(\theta_{n}^{*}(1)\left(\mathcal{L} \mathcal{R}_{u}+\epsilon_{\gamma_{\mathbb{Q}}}^{n} \mathcal{L}_{\infty, p}^{n}\right)\right)\right)^{+}=R_{n}^{+} /\left(\theta_{n}^{*}(1) \cdot \epsilon_{\gamma_{\mathbb{Q}}}^{n} \mathcal{L}_{\infty, p}^{n}\right) .
$$

This completes the proof of Theorem 2.17(ii).

LEMma 6.5. Assume $p$ does not divide $h_{\mathbb{Q}\left(\zeta_{p}\right)^{+}}$and fix an integer $i$ that satisfies $0 \leq i \leq p-2$. Then the group $B_{n \text {,tor }}^{(i)}=A_{n, \text { tor }}^{(i)}$ vanishes if either $i$ is odd or if both $i$ is even and the generalised Bernoulli number $B_{1, \omega^{i-1}}$ is not divisible by $p$. In all other cases this group is a non-trivial cyclic $R_{n}^{(i)}$-module.

Proof. Lemma 3.3(iii) induces an isomorphism of $R_{1}^{(i)}$-modules $H_{0}\left(P_{n}, B_{n}^{(i)}\right) \cong$ $B_{1}^{(i)}$. Since the $R_{n}^{(i)}$-module $\overline{B_{n}^{(i)}}$ is projective (by Theorem 2.17(i)) this isomorphism restricts to give an isomorphism

$$
H_{0}\left(P_{n}, A_{n, \text { tor }}^{(i)}\right)=H_{0}\left(P_{n}, B_{n, \text { tor }}^{(i)}\right) \cong B_{1, \text { tor }}^{(i)}=A_{1, \text { tor }}^{(i)} .
$$

Writing $I_{P_{n}}$ for the augmentation ideal of $\mathbb{Z}_{p}\left[P_{n}\right]$ the Jacobson radical $\operatorname{Jac}\left(R_{n}^{(i)}\right)$ of $R_{n}^{(i)}$ is equal to $p \cdot R_{n}^{(i)}+I_{P_{n}} \cdot R_{n}^{(i)}$ and $R_{n}^{(i)} / \operatorname{Jac}\left(R_{n}^{(i)}\right)$ identifies with $\mathbb{F}_{p}$. Since (22) induces an isomorphism $A_{n, \text { tor }}^{(i)} /\left(\operatorname{Jac}\left(R_{n}^{(i)}\right) \cdot A_{n, \text { tor }}^{(i)} \cong A_{1, \text { tor }}^{(i)} / p\right.$ Nakayama's Lemma therefore implies the minimal number of generators of the $R_{n}^{(i)}$-module $A_{n, \text { tor }}^{(i)}$ is $\operatorname{dim}_{\mathbb{F}_{p}}\left(A_{1, \text { tor }}^{(i)} / p\right)$. We now compute that $\operatorname{dim}_{\mathbb{F}_{p}}\left(A_{1, \text { tor }}^{(i)} / p\right)$ is equal to

$$
\begin{aligned}
& \operatorname{dim}_{\mathbb{F}_{p}}\left(A_{1}^{(i)} / p\right)-\operatorname{dim}_{\mathbb{F}_{p}}\left(\overline{A_{1}^{(i)}} / p\right) \\
= & \operatorname{dim}_{\mathbb{F}_{p}}\left(\left(H_{L_{1}}\left(L_{1}^{\times}\right)^{p} /\left(L_{1}^{\times}\right)^{p}\right)^{(1-i)}\right)-\operatorname{dim}_{\mathbb{F}_{p}}\left(\overline{B_{1}^{(i)}} / p\right)-\operatorname{dim}_{\mathbb{F}_{p}}\left(\mathbb{Z}_{p}^{(i)} / p\right)
\end{aligned}
$$




$$
\begin{gathered}
\left.=\operatorname{dim}_{\mathbb{F}_{p}}\left(\left(P_{L_{1}} / P_{L_{1}}^{p}\right)^{(1-i)}\right)+\operatorname{dim}_{\mathbb{F}_{p}}\left(\mathrm{Cl}_{L_{1}}^{(1-i)} / p\right)-\operatorname{dim}_{\mathbb{F}_{p}} \overline{\left(B_{1}^{(i)}\right.} / p\right)-\operatorname{dim}_{\mathbb{F}_{p}}\left(\mathbb{Z}_{p}^{(i)} / p\right) \\
=\operatorname{dim}_{\mathbb{F}_{p}}\left(\left\langle\zeta_{p}\right\rangle^{(1-i)}\right)+\operatorname{dim}_{\mathbb{F}_{p}}\left(\left(\mathcal{O}_{L_{1}^{+}}^{\times} /\left(\mathcal{O}_{L_{1}^{+}}^{\times}\right)^{p}\right)^{(1-i)}\right)+\operatorname{dim}_{\mathbb{F}_{p}}\left(\left(\mathbb{Z}_{p} / p\right)^{(1-i)}\right) \\
+\operatorname{dim}_{\mathbb{F}_{p}}\left(\mathrm{Cl}_{L_{1}}^{(1-i)} / p\right)-\operatorname{dim}_{\mathbb{F}_{p}}\left(\overline{B_{1}^{(i)}} / p\right)-\operatorname{dim}_{\mathbb{F}_{p}}\left(\mathbb{Z}_{p}^{(i)} / p\right) .
\end{gathered}
$$

Here the first equality is true as Kummer theory combines with the definition of $H_{L_{1}}$ (just prior to (13)) to give an isomorphism $A_{1} / p \cong \operatorname{Hom}\left(H_{L_{1}}\left(L_{1}^{\times}\right)^{p} /\left(L_{1}^{\times}\right)^{p},\left\langle\zeta_{p}\right\rangle\right)$ of $\mathbb{Z}_{p}\left[G_{1}\right]$ modules and because the tautological exact sequence of $\mathbb{Z}_{p}\left[G_{1}\right]$-modules $0 \rightarrow B_{1} \rightarrow A_{1} \rightarrow$ $\Gamma_{L_{1}} \rightarrow 0$ splits. The second equality follows by taking $\omega^{1-i}$-isotypic components of the exact sequence (13) and the last equality is derived easily from the following facts: since the unique prime ideal of $L_{1}$ above $p$ is principal there is an exact sequence of $G_{1}$-modules $0 \rightarrow \mathcal{O}_{L_{1}}^{\times} \rightarrow P_{L_{1}} \rightarrow \mathbb{Z} \rightarrow 0$ and, since $\mathcal{O}_{L_{1}}^{\times}=\mathcal{O}_{L_{1}^{+}}^{\times} \cdot\left\langle\zeta_{p}\right\rangle$ (by [30, Proposition 1.5]) there is a direct sum decomposition of $\mathbb{F}_{p}\left[G_{1}\right]$-modules $\mathcal{O}_{L_{1}}^{\times} /\left(\mathcal{O}_{L_{1}}^{\times}\right)^{p}=\mathcal{O}_{L_{1}^{+}}^{\times} /\left(\mathcal{O}_{L_{1}^{+}}^{\times}\right)^{p} \oplus\left\langle\zeta_{p}\right\rangle$.

Next we note that $\operatorname{dim}_{\mathbb{F}_{p}}\left(\left\langle\zeta_{p}\right\rangle^{(1-i)}\right)$ and $\operatorname{dim}_{\mathbb{F}_{p}}\left(\mathbb{Z}_{p}^{(i)} / p\right)$ are both equal to 1 if $i=0$ and to 0 otherwise, that $[\mathbf{3 0}$, Proposition 8.13$] \operatorname{implies} \operatorname{dim}_{\mathbb{F}_{p}}\left(\left(\mathcal{O}_{L_{1}^{+}}^{\times} /\left(\mathcal{O}_{L_{1}^{+}}^{\times}\right)^{p}\right)^{(1-i)}\right)$ is equal to 1 if $i$ is both odd and bigger than 1 and to zero otherwise, that $\operatorname{dim}_{\mathbb{F}_{p}}\left(\left(\mathbb{Z}_{p} / p\right)^{(1-i)}\right)$ is equal to 1 if $i=1$ and to 0 otherwise, that [30, Corollary 10.15] implies $\operatorname{dim}_{\mathbb{F}_{p}}\left(\mathrm{Cl}_{L_{1}}^{(1-i)} / p\right)$ is equal to 1 if $i$ is even and such that $B_{1, \omega^{i-1}}$ is divisible by $p$ and to 0 otherwise, and that Theorem 2.17(i) implies $\operatorname{dim}_{\mathbb{F}_{p}}\left(\overline{B_{1}^{(i)}} / p\right)$ is equal to 1 if $i$ is odd and to 0 otherwise.

Upon substituting these facts into the above displayed formula for $\operatorname{dim}_{\mathbb{F}_{p}}\left(A_{1, \text { tor }}^{(i)} / p\right)$ one finds that the minimal number of generators of the $R_{n}^{(i)}$-module $A_{n, \text { tor }}^{(i)}$ is

$$
\operatorname{dim}_{\mathbb{F}_{p}}\left(A_{1, \text { tor }}^{(i)} / p\right)= \begin{cases}1, & \text { if } i \text { is even and } p \text { divides } B_{1, \omega^{i-1}} \\ 0, & \text { otherwise }\end{cases}
$$

as required.

\subsection{The proof of Theorem 2.17 (iii).}

In this section we fix an even integer $i$ with $0 \leq i \leq p-3$.

To prove the first assertion of Theorem 2.17 (iii)(a) we assume $p$ divides $B_{1, \omega^{i-1}}$. In this case Lemma 6.5 implies $A_{1, \text { tor }}^{(i)} \neq 0$ and then the surjection $(22)$ implies $A_{n, \text { tor }}^{(i)} \neq$ 0. Since Proposition 6.2 implies $A_{n, \text { tor }}^{(i)}$ is a cohomologically-trivial $P_{n}$-module we can therefore apply Lemma 3.7 to deduce $\left|A_{n, \text { tor }}^{(i)}\right| \geq p^{\Delta_{P_{n}}}$ and since $\Delta_{P_{n}}=n$ (as $P_{n}$ is cyclic of order $p^{n-1}$ ) this proves the first assertion of claim (a).

The first assertion of Theorem 2.17(iii)(b) follows immediately from Lemma 6.5.

To prove all remaining assertions of Theorem 2.17(iii) we first note that Lemma 6.5 combines with the displayed isomorphism in Theorem 2.17(ii) to imply that the element $e_{(i)} \theta_{n}^{*}(1) \cdot \epsilon_{\gamma_{\mathbb{Q}}}^{n} \mathcal{L}_{\infty, p}^{n}$ belongs to $R_{n}^{(i) \times}$ if $p$ does not divide $B_{1, \omega^{i-1}}$ and to $R_{n}^{(i)} \backslash R_{n}^{(i) \times}$ if $p$ divides $B_{1, \omega^{i-1}}$.

We also note that $R_{n}^{(i)}$ is isomorphic to the ring $\mathbb{Z}_{p}\left[P_{n}\right]$ and that for each $\psi$ in $P_{n}^{*}$ one has $\left(e_{(i)} \theta_{n}^{*}(1) \cdot \epsilon_{\gamma_{\mathbb{Q}}}^{n} \mathcal{L}_{\infty, p}^{n}\right)^{\psi}=\epsilon_{\gamma_{\mathbb{Q}}}^{\psi \omega^{i}} \mathcal{L}_{\infty, p}^{\psi \omega^{i}} \cdot L^{*}\left(\psi \omega^{i}, 1\right)$. 
Given these facts, all remaining assertions of Theorem 2.17(iii) are obtained directly by applying the following result with $\Gamma=P_{n}$ and $x=e_{(i)} \theta_{n}^{*}(1) \cdot \epsilon_{\gamma_{\mathbb{Q}}}^{n} \mathcal{L}_{\infty, p}^{n}$.

Lemma 6.6. Let $\Gamma$ be a finite abelian p-group and fix $x$ in $\mathbb{C}_{p}[\Gamma]$.

(i) $x$ belongs to $\mathbb{Z}_{p}[\Gamma]$ if and only if for every element $\gamma$ of $\Gamma$ the congruence

$$
\sum_{\psi \in \Gamma^{*}} \psi(\gamma) x^{\psi} \equiv 0\left(\bmod |\Gamma| \cdot \mathbb{Z}_{p}\right)
$$

is valid in $\mathbb{C}_{p}$.

(ii) If $x$ belongs to $\mathbb{Z}_{p}[\Gamma]$, then $x$ belongs to $\mathbb{Z}_{p}[\Gamma] \backslash \mathbb{Z}_{p}[\Gamma]^{\times}$, resp. to $\mathbb{Z}_{p}[\Gamma]^{\times}$, if and only if $x^{\mathbf{1}_{\Gamma}}$ belongs to $p \cdot \mathbb{Z}_{p}$, resp. to $\mathbb{Z}_{p}^{\times}$.

Proof. To prove claim (i) note that

$$
x=\sum_{\psi \in \Gamma^{*}} x^{\psi} e_{\psi}=\sum_{\psi \in \Gamma^{*}} x^{\psi}|\Gamma|^{-1} \sum_{\gamma \in \Gamma} \psi(\gamma) \gamma^{-1}=\sum_{\gamma \in \Gamma}|\Gamma|^{-1}\left(\sum_{\psi \in \Gamma^{*}} \psi(\gamma) x^{\psi}\right) \gamma^{-1}
$$

and hence that $x$ belongs to $\mathbb{Z}_{p}[\Gamma]$ if and only if for every element $\gamma$ of $\Gamma$ the sum $\sum_{\psi \in \Gamma^{*}} \psi(\gamma) x^{\psi}$ belongs to $|\Gamma| \cdot \mathbb{Z}_{p}$.

We note next that $\mathbb{Z}_{p}[\Gamma]$ is a local ring with maximal ideal equal to the set of elements $x=\sum_{\gamma \in \Gamma} x_{\gamma} \gamma$, with each $x_{\gamma}$ in $\mathbb{Z}_{p}$, such that $\sum_{\gamma \in \Gamma} x_{\gamma}$ belongs to $p \cdot \mathbb{Z}_{p}$. This implies claim (ii) because

$$
\sum_{\gamma \in \Gamma} x_{\gamma}=\sum_{\gamma \in \Gamma} \sum_{\psi \in \Gamma^{*}}|\Gamma|^{-1} \psi(\gamma) x^{\psi}=\sum_{\psi \in \Gamma^{*}}|\Gamma|^{-1}\left(\sum_{\gamma \in \Gamma} \psi(\gamma)\right) x^{\psi}=x^{\mathbf{1}_{\Gamma}}
$$

\subsection{The proof of Theorem 2.17 (iv).}

Fix an odd integer $i$ with $1 \leq i \leq p-3$.

In this case Lemma 6.5 implies $A_{n \text {,tor }}^{(i)}$ vanishes. This implies that Fit $_{R_{n}}\left(A_{n, \text { tor }}\right)^{(i)}$ $=\operatorname{Fit}_{R_{n}^{(i)}}\left(A_{n, \text { tor }}^{(i)}\right)$ is equal to $R_{n}^{(i)}$ and hence, via the equality of Theorem 6.4 , that $e_{(i)} \theta_{n}^{*}(1)\left(\mathcal{L R}_{u}+\epsilon_{\gamma_{\mathbb{Q}}}^{n} \mathcal{L}_{\infty, p}^{n}\right)=e_{(i)} \theta_{n}^{*}(1) \cdot \mathcal{L} \mathcal{R}_{u}$ is a unit of the ring $R_{n}^{(i)}$.

This fact implies Theorem 2.17(iv) via a simple application of Lemma 6.6 (in just the same way that it was used to prove the second assertion of Theorem 2.17(iii)(b) and the corresponding case of the congruences in Theorem 2.17(iii)(c)).

This completes the proof of Theorem 2.17 .

\section{References}

[1] F. Bertrandias and J.-J. Payan, Г-extensions et invariants cyclotomiques, Ann. Sci. École Norm. Sup., 5 (1972), 517-543.

[2] M. Breuning and D. Burns, On Equivariant Dedekind Zeta-Functions at $s=1$, Documenta Math., Extra Volume: Andrei A. Suslin's Sixtieth Birthday, 2010, 119-146.

[ 3 ] K. S. Brown, Cohomology of groups, Graduate Texts in Math., 87, Springer, New York, 1992.

[4] A. Brumer, On the units of algebraic number fields, Mathematika, 14 (1967), 121-124.

[5] D. Burns, On the Galois structure of arithmetic cohomology I: compactly supported $p$-adic cohomology, submitted for publication, to appear in Nagoya Math. J. 
[6 ] D. Burns, On the Galois structure of arithmetic cohomology III: Selmer groups of critical motives, to appear in Kyoto J. Math.

[ 7 ] D. Burns and M. Flach, Equivariant Tamagawa numbers for motives with (non-commutative) coefficients, Doc. Math., 6 (2001), 501-570.

[ 8 ] D. Burns and D. Macias Castillo, On $p$-adic $L$-series, $p$-adic cohomology and class field theory, J. Reine Angew. Math., 732 (2017), 55-84.

[9] H. Castillo and A. Jones, On the values of Dedekind Zeta functions at $s=1$ and annihilation of class groups, Acta Arith., 160 (2013), 67-93.

[10] F. E. Diederichsen, Über die Ausreduktion ganzahliger Gruppendarstellungen bei arithmetischer Aquivalenz, Abh. Math. Sem. Univ. Hamburg, 13 (1939), 357-412.

[11] G. G. Elder, On Galois structure of the integers in cyclic extensions of local number fields, J. Théor. Nombres Bordeaux, 14 (2002), 113-149.

[12] M. Flach, Euler characteristics in relative K-groups, Bull. London Math. Soc., 32 (2000), $272-284$.

[13] G. Gras, Groupe de Galois de la $p$-extension abélienne $p$-ramifiée maximale d'un corps de nombres, J. Reine Angew. Math., 333 (1982), 86-132.

[14] A. Heller and I. Reiner, Representations of cyclic groups in rings of integers, I, Ann. of Math., 76 (1962), 73-92.

[15] A. Heller and I. Reiner, Representations of cyclic groups in rings of integers, II, Ann. of Math., 77 (1963), 318-328.

[16] D. Hemard, Modules galoisiennes de torsion et plongements dans les $\mathbb{Z}_{p}$-extensions, J. Number Theory, 30 (1988), 357-374.

[17] J-F. Jaulent and T. Nguyen Quang Do, Corps p-rationnels, corps $p$-réguliers et ramification restreinte, J. Théor. Nombres Bordeaux, 5 (1993), 343-363.

[18] C. Khare and J-P. Wintenberger, Ramification in Iwasawa Theory and Splitting Conjectures, Int. Math. Res. Notices, 2014 (2014), 194-223.

[19] Y. Kida, $\ell$-Extensions of CM-Fields and Cyclotomic Invariants, J. Number Theory, 12 (1980), 519-528.

[20] F. Knudsen and D. Mumford, The projectivity of the moduli space of stable curves I: Preliminaries on 'det' and 'Div', Math. Scand., 39 (1976), 19-55.

[21] H. Miki, On the Leopoldt Conjecture on the $p$-adic regulators, J. Number Theory, 26 (1987), $117-128$.

[22] H. Miki and H. Sato, Leopoldt's conjecture and Reiner's theorem, J. Math. Soc. Japan, 36 (1984), 47-52.

[23] A. Movahhedi and T. Nguyen Quang Do, Sur l'arithmétique des corps de nombres p-rationnels, Sém. Th. Nombres Paris 1987/1988, Prog. in Math., 89 (1990), 155-200.

[24] J. Neukirch, A. Schmidt and K. Wingberg, Cohomology of number fields, Springer-Verlag, Berlin, 2000 .

[25] B. Oriat, Annulation de groupes de classes reelles, Nagoya Math. J., 81 (1981), 45-56.

[26] S. Seo, On first layers of $\mathbb{Z}_{p}$-extensions, J. Number Theory, 133 (2013), 4010-4023.

[27] J-P. Serre, Sur le résidue de la fonction zêta $p$-adique d'un corps de nombres, C.R. Acad. Sci. Paris, 278 (1978), 183-188.

[28] I. R. Shafarevic, Extensions with prescribed ramification points, Publ. Math. I.H.E.S., 36 (1986), 71-95.

[29] J. Tate, Les Conjectures de Stark sur les Fonctions $L$ d'Artin en $s=0$ (notes par D. Bernardi et N. Schappacher), Progress in Math., 47, Birkhäuser, Boston, 1984.

[30] L. C. Washington, Introduction to Cyclotomic Fields, Graduate Texts in Math., 83, SpringerVerlag, Berlin, 1982.

[31] A. V. Yakovlev, Homological definability of $p$-adic representations of a ring with power basis, Izvestia A N SSSR, ser. Math., 34 (1970), 321-342 (In Russian).

[32] A. V. Yakovlev, Homological definability of $p$-adic representations of groups with cyclic Sylow p-subgroup, An. St. Univ. Ovidius Constanţa, 4 (1996), 206-221. 


\section{David BURNS}

King's College London

Department of Mathematics

London WC2R 2LS, U.K

E-mail: david.burns@kcl.ac.uk
Asuka KumON

King's College London

Department of Mathematics

London WC2R 2LS, U.K

E-mail: asuka.kumon@kcl.ac.uk 